\title{
Direct Simulation of the Self-Assembly of a Small DNA Origami
}

\author{
Benedict E. K. Snodin, ${ }^{*} \dagger$ Flavio Romano, ${ }^{\dagger}$ Lorenzo Rovigatti, ${ }^{\ddagger}$ Thomas \\ E. Ouldridge, ${ }^{\Uparrow}$ Ard A. Louis, ${ }^{\S}$ and Jonathan P. K. Doye*,† \\ $\dagger$ Physical and Theoretical Chemistry Laboratory, Department of Chemistry, University of \\ Oxford, South Parks Road, Oxford, OX1 3QZ, United Kingdom \\ $\ddagger$ Faculty of Physics, University of Vienna, Boltzmanngasse 5, A-1090 Vienna, Austria \\ IDepartment of Mathematics, Imperial College, 180 Queen's Gate, London SW7 2AZ, \\ United Kingdom \\ $\S$ Rudolf Peierls Centre for Theoretical Physics, University of Oxford, 1 Keble Road, Oxford, \\ OX1 3NP, United Kingdom \\ E-mail: benedict.snodin@chem.ox.ac.uk; jonathan.doye@chem.ox.ac.uk
}

\section{Abstract}

By using oxDNA, a coarse-grained nucleotidelevel model of DNA, we are able to directly simulate the self-assembly of a small 384-base-pair origami from single-stranded scaffold and staple strands in solution. In general, we see attachment of new staple strands occurring in parallel, but with cooperativity evident for the binding of the second domain of a staple if the adjacent junction is already partially formed. For a system with exactly one copy of each staple strand, we observe a complete assembly pathway in an intermediate temperature window; at low temperatures successful assembly is prevented by misbonding while at higher temperature the free-energy barriers to assembly become too large for assembly on our simulation time scales. For high-concentration systems involving a large staple strand excess, we never see complete assembly because there are invariably instances where copies of the same staple both bind to the scaffold, creating a kinetic trap that prevents the complete binding of either staple. This mutual staple blocking could also lead to aggregates of partially formed origamis in real systems, and helps to rationalize certain successful origami design strategies.

\section{Keywords}

self-assembly, DNA nanotechnology, simulation, coarse-grained modelling, DNA origami

\section{Introduction}

DNA nanotechnology is an exciting and rapidly growing field, $1+5$ in which the A-T, G-C specificity of DNA's Watson-Crick base pairing is leveraged to fabricate a wide range of nanostructures and nanodevices. A particularly powerful and now widespread method of creating these structures, named DNA origami, was devised by Rothemund in 2006. ${ }^{6}$ This method of DNA self-assembly involves folding a very long DNA strand (typically around 7.5 kilo-bases), termed the scaffold strand, by the action of many short strands, termed staple strands. Each staple strand binds to two or more nonadjacent regions of the scaffold strand, pulling them close together in order to form the target structure. Initially, this method was used to generate 2D structures composed of antiparallel double helices joined by crossovers, where staple strands cross from one region of the scaffold to another. Subsequently, the method was used 
to create $3 \mathrm{D}$ shapes, ${ }^{7}$ including structures with a designed curve or twist, $\underline{89}$ as well as tensegrity structures. 10

The ease and reliability of assembly, flexibility of design and addressability of DNA origami structures opens up a variety of practical applications, which are only beginning to be explored. A common use is as a substrate for single-molecule studies, $\underline{11}$ for example to probe hairpin dynamics ${ }^{12}$ or the motion of walkers. 13 Other promising applications include use as drug delivery vehicles, 1415 as artificial membrane channels $\frac{16] 17}{10}$ and nanopores, $\frac{18}{1}$ and as stiff handles for single-molecule mechanical experiments with optical tweezers. $\frac{19}{19}$

The wide ranging potential applications of DNA origami have motivated efforts to systematically understand the structure and selfassembly of these DNA origami objects. Their detailed structural properties have been revealed by cryo-electron microscopy, 20 and have been investigated computationally with allatom simulations ${ }^{21}$ and with models at a much more coarse-grained level.22] 25$]$ There have also been attempts to better understand their selfassembly through experiment. ${ }^{26}$ In a careful study, Sobczak et al. ${ }^{[27}$ probed the assembly of certain 2D and 3D origami structures, and found rapid assembly in a narrow temperature window for each structure. Further work from the same group ${ }^{28}$ attempted to quantify folding quality in origami assembly, by binding fluorescent markers to any single-stranded regions of the scaffold strand. Similarly, Ke and coworkers ${ }^{29}$ examined the effect of staple-break positioning on assembly and found that the yield could be very sensitive to this aspect of the design. Very recently, Dunn et al. designed a "dimer" origami that had multiple fully-bonded states, and thus allowed folding pathways to be inferred from the assembly products. ${ }^{30}$ The experimental work was complemented with a domain-level kinetic model ${ }^{31}$ (which extended and improved upon previous such models ${ }^{30 \mid 32}$ ) whose predictions were in good agreement with the experimental results, implying that the model predicted a realistic set of folding pathways.

In Ref. 33, Cademartiri and Bishop provided an interesting classification scheme that divides self-assembly into two types, namely a "puzzle" limit in which the assembly materials are separate units, each carrying information about where they should fit in the final structure through specific interactions (e.g. "DNA bricks" 34 ); and a "polymer" limit in which a polymer made of a limited number of repeating units folds up, with the topology of the polymer and weak, non-specific interactions between the polymer units determining the final structure (e.g. protein folding). DNA origami self-assembly can be regarded as a mixture of the two, with a long scaffold strand, akin to the polymer limit, which folds by the binding of many short staple strands to specific domains of the scaffold, similar to the puzzle limit. Despite the research efforts described earlier, important open questions remain regarding this mixedtype assembly. In particular, it is not clear whether the assembly is a "nucleated" process (i.e. one with a free-energy barrier), nor how and why certain changes to the origami design lead to different assembly yields.

Simulations can potentially provide insight into the self-assembly process through visualization of the assembly mechanism in a way not possible in experiment. However, all-atom approaches are ruled out as the time and length scales relevant for origami self-assembly are far beyond those that can realistically be achieved with today's computers. ${ }^{35}$ A potential way forward is provided by coarse-grained DNA models, as their simplified representations of DNA allow access to much longer time and length scales. In order to be useful, such models must capture the physics of DNA relevant to the assembly while still being simple enough to permit efficient computation.

The oxDNA model ${ }^{36}$ satisfies both of these requirements. oxDNA has proven to be a robust and predictive model of DNA, $\stackrel{37}{3}$ and is being applied to an increasing number of DNA nanotechnology systems. 38 Importantly for this study, it captures the biophysics of DNA likely to be relevant for origami self-assembly, such as hybridization, $\underline{41}$ displacement, $\underline{42143}$ and the conformational properties of single-stranded DNA. ${ }^{36}$ Furthermore, with a GPU (graphical 
processing unit) version of the code implementing oxDNA it is possible to efficiently simulate systems consisting of thousands of nucleotides. 44

In this work we use this model to investigate the self-assembly of DNA origami. We do this by attempting to simulate directly the assembly of a small model origami structure starting from its single-stranded components. For this origami, we are able to identify kinetic traps that hinder self-assembly as well as conditions under which we observe complete assembly. Although computational constraints limit us to considering a small origami design (384 base pairs rather than the usual $\sim 7000$ ), this still represents by far the largest DNA nanostructure whose self-assembly has been directly simulated. Furthermore, we are confident that many of the insights we obtain are applicable to full-scale origamis.

\section{Results and Discussion}

System and model. oxDNA, a coarse-grained model of DNA. In oxDNA, a strand is represented by a chain of rigid bodies, with each rigid body corresponding to a nucleotide. The nucleotides interact through an effective potential designed to capture hydrogen bonding between base pairs, stacking, backbone connectivity and excluded volume interactions. The stacking and hydrogen-bonding terms have a complex form with angular dependence, to capture the geometric constraints of the interactions in DNA. There is no explicit solvent, with the effect of the solvent being incorporated into the interactions between the nucleotides and into the thermostat. The potential is fitted to reproduce the experimentally-determined structure and mechanics of single- and double-stranded DNA as well as the thermodynamics of hybridization. Further details are given in Ref. 36 .

The parameterization of the oxDNA model we use here is an "average-base" one in which the strength of the stacking and Watson-Crick base-pairing interactions are independent of the identity of the bases. We use this parameterization, rather than a sequence-dependent one, ${ }^{45}$ because we are interested in the generic properties of the self-assembly rather than the specifics of how it depends on sequence. We also note that the only base-pairing interactions allowed in the model are of the Watson-Crick type and only occur between complementary bases; for example, Hoogsteen base pairs are not possible in oxDNA. However, this simplification should not affect our results as such nonWatson-Crick interactions are unlikely to be relevant at the high temperatures where assembly takes place. The oxDNA model is parameterized to a salt concentration of $0.5 \mathrm{M}$, which is representative of the high salt conditions typically used for origami assembly where the electrostatic interactions are largely screened. We also note that an updated version of oxDNA, called oxDNA2, was recently released. ${ }^{46}$ Here, we use the original model as the extremely long simulations presented here were started well before the release of the new model. Moreover, the improvements in the new model are unlikely to be important for the self-assembly behaviour that is the focus here, and so the two models would be expected to give very similar results.

To simulate origami self-assembly, we use molecular dynamics (MD) coupled to an Andersen thermostat, which approximates the Brownian motion of DNA in solution. Even at our level of coarse graining, the simulations presented here are computationally very demanding: each of the runs presented required several months of computer time on a GPU.

Assembly of a small origami. In this work, we study the assembly of a small, 384-base-pair origami from single strands in solution. The design of the origami is shown in Fig. 1. Note that the helices in this structure are not perfectly parallel. This is because it is favourable for the helices to bend away from each other at the junctions, which leads to the weave pattern that is also seen in micrographs of DNA origami structures. In our example the relatively small size of the origami and the low number of constraining junctions leads to a further splaying out of the helices.

The scaffold strand can be divided up into 24 16-base domains (labelled 1-24 in Fig. 1 starting from the $3^{\prime}$ end) with each staple strand 
binding to two of these domains. There are twelve different staples, which can be divided into four types (Fig. 1). There are two "end" ("E") staples that bind to two consecutive domains at the two ends of the scaffold in a single double helix. The rest have two double-helical domains, and one staple crossing, where we use staple crossing to refer to the point at which the staple crosses from one region of the scaffold to another, folding the scaffold into the target shape. The non-E staples are classified as "L", "M" and "R", corresponding to whether they are on the left, middle or right of the origami when oriented as in Fig. 1. In this design, there are two types of four-way junction: one type involving the $\mathrm{M}$ and $\mathrm{L}$ staples, which has two staple crossings at the junction, and the other type having just a single staple crossing from one of the $\mathrm{R}$ staples.

In order to make origami self-assembly more feasible on our computational time scales, we designed the DNA strands' sequences to minimize secondary-structure formation in the single strands, as it is known that hairpins can significantly slow down the rate of duplex formation. ${ }^{4849}$ One consequence of this choice is that misbonding between scaffold and staple strands is somewhat more likely than for random sequences. All the sequences are given in Table S2 in the Supporting Information.

In this work we consider assembly under two different concentration ratios between the staples and scaffold: firstly, we consider a system with a large excess of staples (namely 17-fold) and secondly, a system with a stoichiometric mixture (i.e. only one copy of every staple in our simulation).

In our simulations, we consider the assembly of a single origami, i.e. we have only one scaffold strand in our simulation box. We use a scaffold strand concentration of $7.7 \mu \mathrm{M}$, and staple strand concentrations of $7.7 \mu \mathrm{M}$ and $130.9 \mu \mathrm{M}$ for the stoichiometric and excess-staple system, respectively. These conditions correspond to an approximately $1000 \times$ higher concentration than typical experimental concentrations (for example, Dietz and coworkers ${ }^{\sqrt{8}}$ used a scaffold strand concentration of $20 \mathrm{nM}$ to assemble 3D origamis). Without such a high strand con-

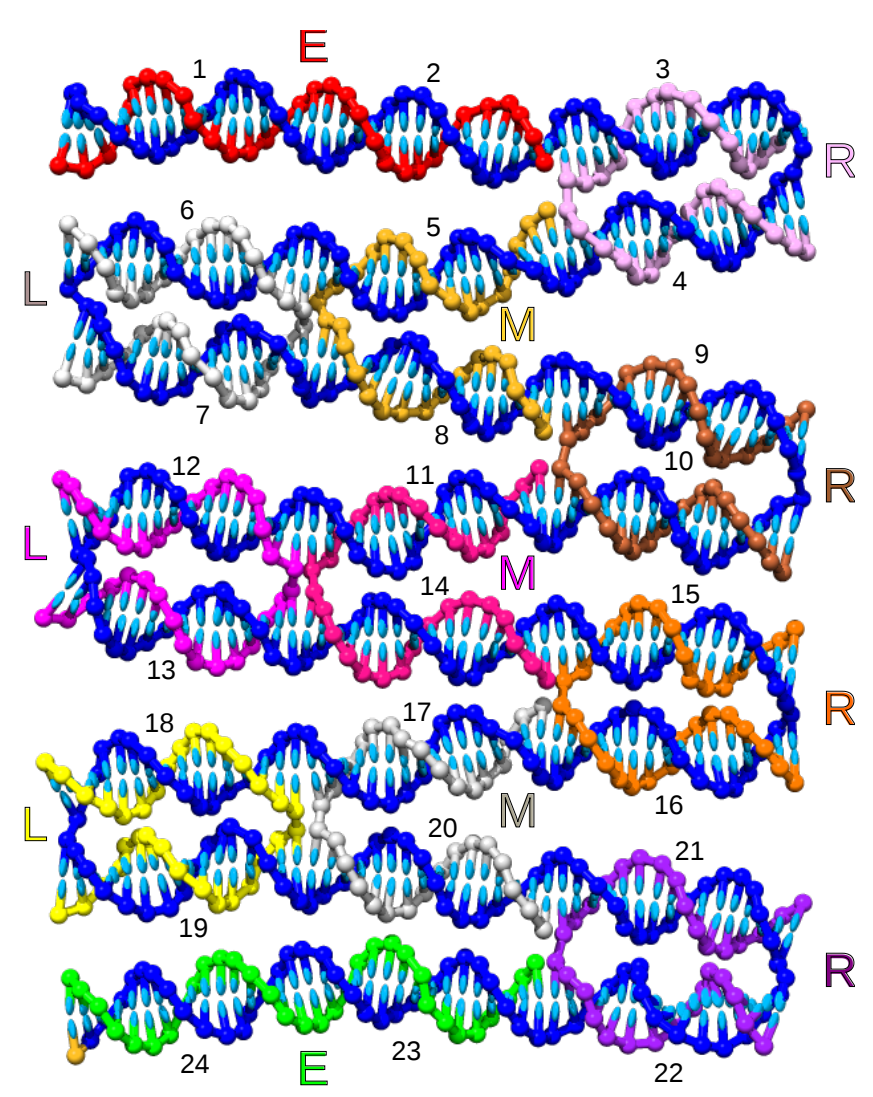

Figure 1: The design of the complete origami. The 384-base scaffold strand is blue (with the $5^{\prime}$ nucleotide gold), while each of the twelve 32base staple strands are depicted in a unique colour, in a colour scheme which is maintained throughout this work. The scaffold is divided up into 24 binding domains, and the staples are labelled according to their type, "E", "R", "M" and "L", according to their general position in the complete origami structure. As the complete origami is very unlikely to have a planar structure in solution, in order to generate a configuration where the design of the origami is clear, a simulation was run in which we explicity added an attractive surface onto which the origami adsorbed. Configurations in this and subsequent figures have been visualized using the USCF Chimera package. $\stackrel{47}{ }$ 
centration it would not be feasible to observe self-assembly in our simulations, as almost all the simulation time would be spent waiting for each of the staple strands to find their scaffold domains by diffusing around an enormous simulation box.

It is likely that there is a relatively narrow temperature window for optimal assembly, as evidenced in recent experiments. ${ }^{27}$ If the temperature is too low secondary structure in the staples and scaffold can occur, as well as misbonding (that is, Watson-Crick base pairing between bases that are not paired in the target structure) between staples and scaffold. Both these effects may result in kinetic traps which will slow down or even prevent correct assembly. The window will be bounded from above by the melting temperature of the origami, but even below this temperature assembly may be hampered by large nucleation free-energy barriers, a slower attachment rate and an increased rate of melting for partially or fully bound staples.

These latter effects are particularly problematic for the direct simulation of origami assembly. In practice, it may only be possible to see assembly in our simulations on reasonable time scales when the correct binding of a staple to a domain is relatively irreversible, placing our simulations of assembly at the lower end of (or perhaps below, where kinetic trapping is relevant) this optimal temperature window. An important reference temperature in this regard is the oxDNA melting temperature for a single 16-base-pair duplex $\left(T_{16 \text { mer }}=66^{\circ} \mathrm{C}\right.$ and $72{ }^{\circ} \mathrm{C}$ for the stoichiometric and excess-staple assembly DNA strand concentrations, respectively) and so we generally chose to run our simulations close to these temperatures.

To summarize the results of our self-assembly simulations we use kymographs, such as the one illustrated in Fig. 2, which show the state of each of the 24 scaffold domains as a function of time. We designate each base pair between each domain and any staples that have fully or partially bound to it as belonging to one of three categories: (i) native (without blocking), indicating a native base pair, i.e. one which is present in the target structure, in the absence of any blocking (see below) (ii) misbonded, indicating a non-native base pair, i.e. one which is not present in the target structure, and (iii) native (with blocking), indicating a native base pair, but one where rather than one staple strand binding to both its scaffold domains, two copies of the same staple strand each bind to only one of the scaffold domains, preventing either of them from reaching their correct fullybound state until one of them melts away or is displaced. Blocking is described in further detail below. Note that it is possible for a scaffold domain to simultaneously experience misbonding and partial native bonding (either blocked or not blocked).

Assembly with a large staple excess: $65^{\circ} \mathrm{C}$. A kymograph for an assembly with an excess of staples and at a temperature of $65^{\circ} \mathrm{C}$ is shown in Fig. 2. Of the four assembly runs simulated under these conditions, this one had the most correctly bound domains by the end of the simulation. From this example it is clear that most of the origami is able to assemble correctly, with 20 of the 24 scaffold domains correctly bound at the end of the simulation (see Fig. 3 for a simulation snapshot). There are a number of important features about the nature of the origami self-assembly that can be gleaned from Fig. 2 .

Local features of staple binding. Although correct binding of a staple to its corresponding domain on the scaffold is a rare event — the two have first to diffuse close enough to interact — when it does occur it does so relatively rapidly, as seen by the step-like increases in the number of native bonds in a domain from zero to approximately 16 . Thus, even at the relatively high concentrations we consider, there is a clear separation between the intermolecular time scales associated with strand diffusion and the intramolecular time scales associated with going from the formation of an initial contact to complete binding of a domain. This behaviour is what we expect based on prior studies of hybridization in oxDNA. $\stackrel{38}{\text { In addition, }}$ correct binding of a 16-base-pair region is effectively irreversible at this temperature on our time scales.

Global features of origami self-assembly. 


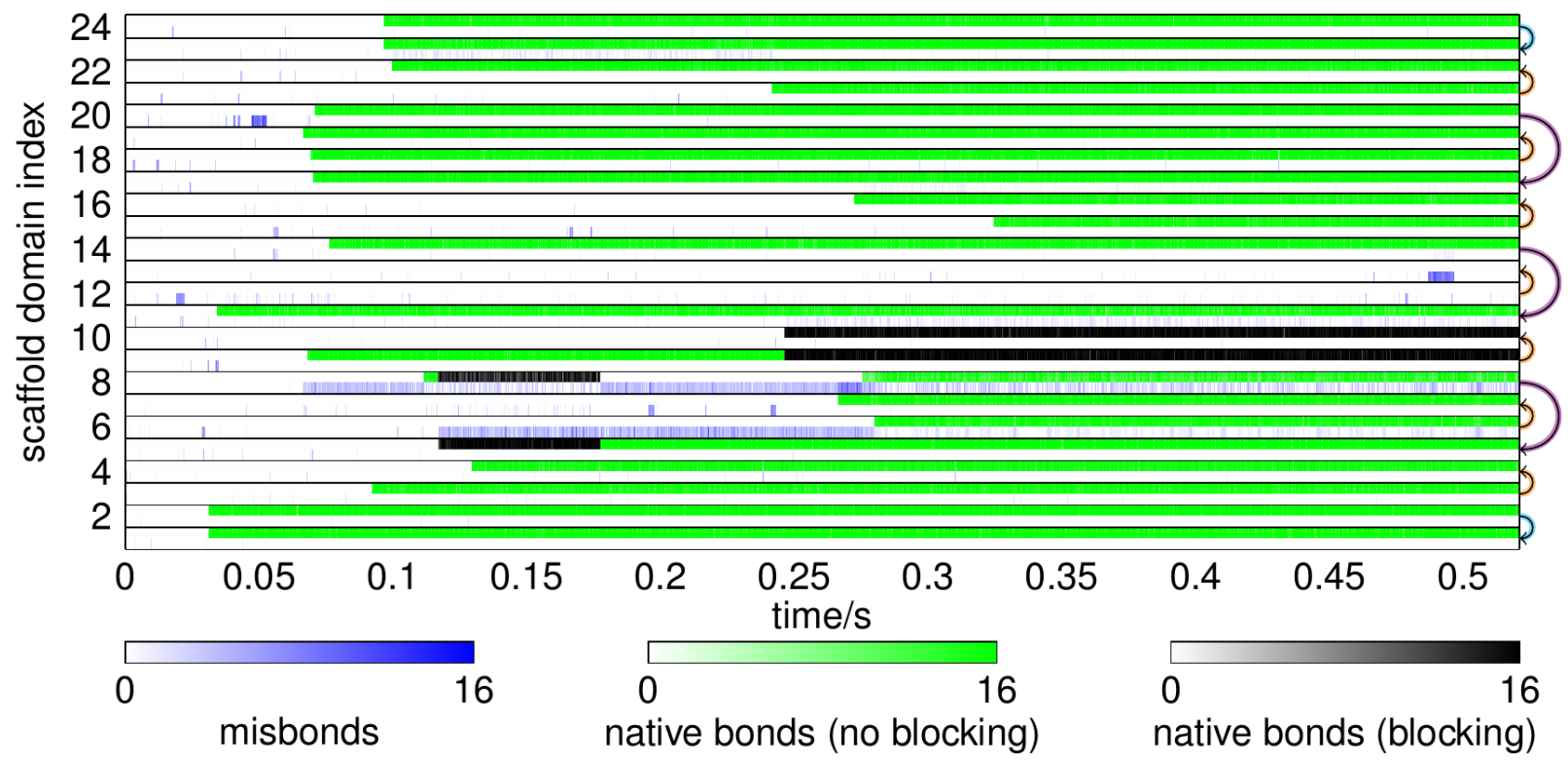

Figure 2: A kymograph at $65^{\circ} \mathrm{C}$ for a single assembly simulation with a $17 \times$ excess of staples, starting in an unbonded configuration. Each row shows the state of a domain of the scaffold strand as a function of time. The top half of each row is either white, red or green. Red and green both indicate native base pairing (i.e. the presence of base pairs which are also found in the target structure) in that domain. Red indicates that the staple bound to that domain is blocked by another staple (see main text), while green indicates that it is not. White indicates no native base pairing. The bottom half of each row is either white or blue, with blue indicating misbonding (i.e. the presence of base pairs which are not found in the target structure) and white indicating no misbonding. In all cases, the intensity of the colour corresponds to the fraction of base pairs within the domain which are in that state. On the right, the curly arrows show the binding pattern of the staple strands, with the arrow head showing where the $5^{\prime}$ end of the staple binds. 


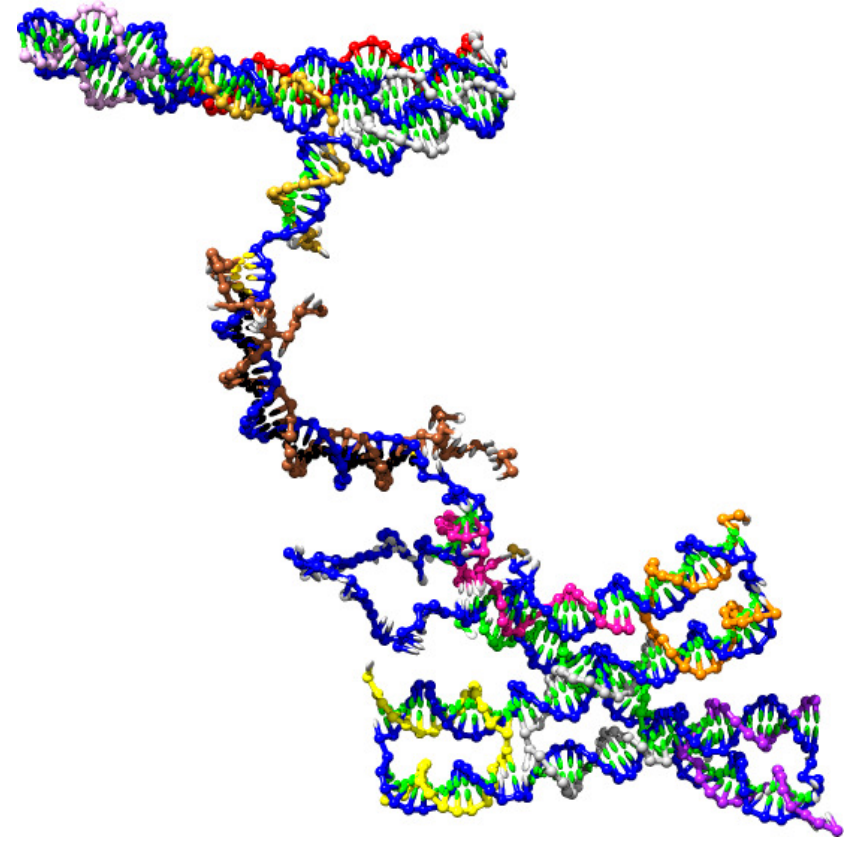

Figure 3: A snapshot from the end of the excess-staple $65^{\circ} \mathrm{C}$ assembly whose kymograph is shown in Fig. 2. The base sites are coloured according to the classification of their base pairing (while the rest of the nucleotide is coloured according to its strand). Unpaired base sites are white, natively-bonded, unblocked base sites are green, misbonded base sites are yellow, and blocked base sites are red.
Firstly, assembly occurs in a relatively parallel manner. In particular, the assembly that is happening in distant sections of the scaffold is relatively independent, as can be seen from the absence of obvious long-range cooperativity in the kymographs for the different assembly runs at $65^{\circ} \mathrm{C}$ (Fig. 2 and S2). This is to be expected given the small number of crossovers in our relatively simple origami design, meaning that the formation of each domain (along with any associated crossovers) only affects other domains that are close to it. Furthermore, the kymographs also suggest that there is no set order in which the staples bind, although this may partly reflect the relatively low temperatures we have chosen. In particular, the initial binding of each staple appears unaffected by both the nearby and distant state of the partially formed origami.

Secondly, there are some signs of cooperativity in the binding of a staple to the second of its scaffold domains, i.e. the binding of the second domain is not necessarily independent of which adjacent domains are occupied by other staples. Generally, binding the second staple domain can be relatively slow because the relevant parts of the staple-scaffold complex have to diffuse close enough to allow binding. For example, in Fig. 2 a staple correctly binds to scaffold domain 22 after around $0.1 \mathrm{~s}$, but only manages to bind to scaffold domain 21 after a further $0.15 \mathrm{~s}$. Note that these time scales would be better separated for the lower concentrations typical of experimental origami assembly; at these concentrations the binding of the first domain would be much slower while the rate of binding for the second domain would be unchanged. 31

For the four scaffold domains adjacent to each of the double-crossover junctions (which are bound by an L staple and an M staple), we see a much faster binding of the fourth domain than is typical for an equivalent binding elsewhere in the assembly. This is likely to be because the scaffold strand is constrained by the first three domains to be in a geometry that makes binding of the fourth domain relatively easy. This can be seen separately for scaffold domains 58 and 17-20 in the kymograph in Fig. 2, and 
simulation snapshots illustrate the process for scaffold domains 17-20 in Fig. 4.

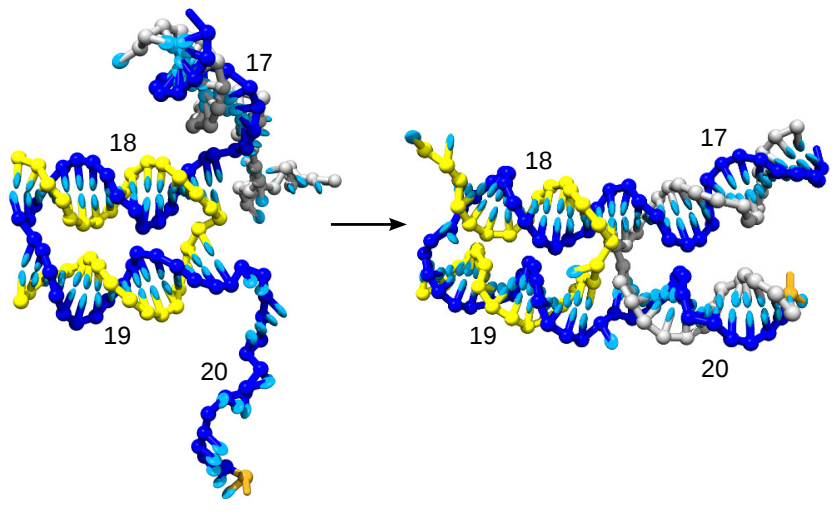

Figure 4: Cooperative binding of a staple to form a double crossover. Only domains 17-20 of the scaffold are depicted. The partially bound grey staple is in a favourable configuration to bind to scaffold domain 20 because of the correctly bound yellow staple.

Barriers to complete assembly. (i) Misbonding. The misbonding observed for the assembly simulations can be divided into two types, the first of which is not much of a hindrance to assembly and the second of which is more disruptive. Firstly, it is possible for a staple strand to bind to the scaffold purely by misbonding. This behaviour can be seen in the kymograph in Fig. 2 as the short stretches of blue scattered throughout the graph. Such events are common in the initial stages of the simulation when most of the scaffold is still available for binding. However, these misbonded configurations only persist for a relatively short amount of time before melting away and, during the time they are present, the number of base pairs fluctuates a lot as the duplexes fray and reform. For these reasons, they offer little resistance to correct assembly of the origami at this temperature.

The second type of misbonding can occur when a staple is correctly bound to one of its scaffold domains. It is then sometimes favourable to extend the double helix beyond the end of this domain as it may be able to form some base pairs with the next domain (for example, see Fig. 5(a)). This type of misbonding is more persistent (see scaffold domains 6 and 8 in Fig. 2), because the strands involved are unlikely to detach from the scaffold due to their correctly bound domain, and is thus potentially more detrimental to the assembly.

This persistent misbonding can interfere with origami assembly in two ways. Firstly, it will be harder for the misbonding scaffold domain to be bound by the correct staple, because the domain is partially blocked by the misbonding staple. Note that this is not too much of a hindrance, because the correct staple still has the possibility of beginning to bind to some of the free scaffold bases, after which it will potentially be able to displace the misbonded staple. For example, in Fig. 2, the correct staple is able to bind to domain 8 at around $0.11 \mathrm{~s}$ despite the misbonding in domain 8 due to the staple bound to domain 9, as illustrated in Fig. 5(a) and Fig. 5(b) (although in this case it happens to melt later in the simulation, probably partly due to misbonding from the domain 9 staple).

Secondly, persistent misbonding makes it harder for the strand that is misbonded to find its own correct second scaffold domain. Again, this only slows down rather than stops assembly, because there is still some of the staple unbound and available (this amount can fluctuate considerably) to bind to the correct domain, although clearly the misbonding will often impose severe geometrical constraints on the staple bases that remain free. For example, in Fig. 2, the staple correctly bound to domain 5 misbonds with domain 6 , but is still able to bind later to domain 8 . Relevant simulation snapshots are shown in Fig. 5(e) and Fig. 5(f).

We should note both that, as mentioned earlier, the sequence we have used makes misbonding more likely than for a random sequence, and that oxDNA tends to overestimate the thermodynamic stability of misbonded states somewhat. ${ }^{36}$ Therefore, we expect that misbonding is less likely to be a problem for real DNA at an equivalent temperature. Nevertheless, in both simulation and experiment, as the temperature is decreased the misbonding that occurs is expected to become more persistent, until at sufficiently low temperature it can be effectively irreversible, and hence prevent complete assembly. This will be explored more when considering stoichiometric assembly, where we have performed simulations at lower temperatures. 


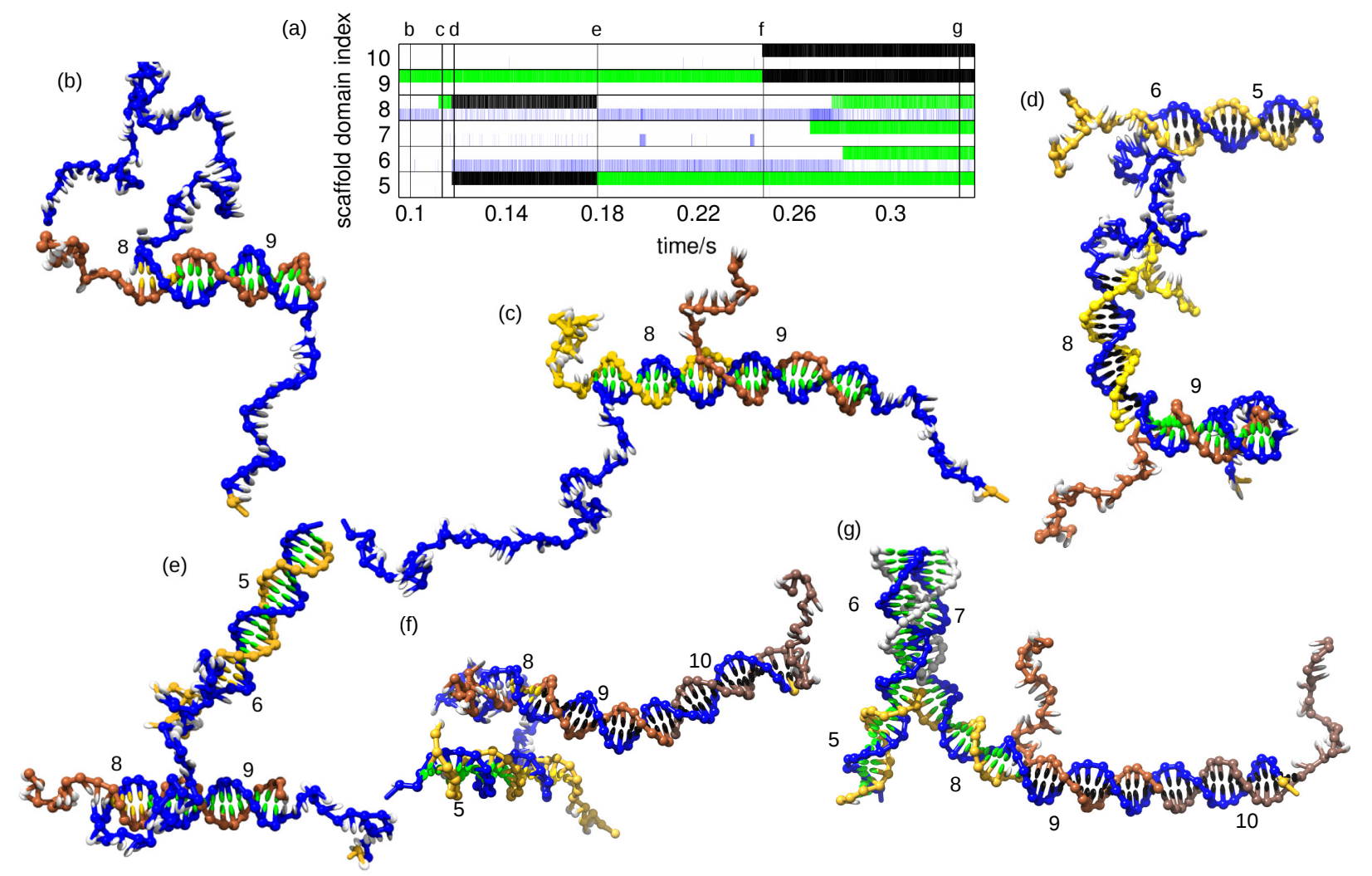

Figure 5: A series of snapshots from the trajectory corresponding to the kymograph in Fig. 2 illustrating misbonding and blocking. Only the scaffold domains 5-10, and any staples with at least one base pair with those scaffold domains, are depicted. Note that some staple domains are bound to a scaffold domain that is not shown. The base sites are coloured white if unpaired, green if natively-bonded and unblocked, yellow if misbonded and red if blocked. (a) An extract from the kymograph in Fig. 2 is shown, with letters along the top showing where the snapshots appear in the kymograph. (b) A staple is natively bound to domain 9 and partially misbonded to domain 8. (c) A correct staple comes in to bind to domain 8, although there is still some misbonding in domain 8 due to the domain 9 staple. (d) Before the domain 8 staple can complete its binding by binding to domain 5, a second copy of the same staple binds to domain 5, as well as partially misbonding to domain 6. (e) The original staple, which bound to domain 8, melts off, partly due to the domain 9 staple misbonding with domain 8. The domain 5 staple is now no longer blocked. (f) A copy of the domain 9 staple comes in and binds to domain 10, blocking the domain 9 staple. (g) A new staple comes in and binds to domains 6 and 7, while the domain 5 staple binds to domain 8, finishing its binding. Although domains 5-8 are correctly bound, the blocking in domains 9 and 10 remains. 
(ii) Blocking. The major obstacle to complete assembly of the origami in our simulations is what we have termed "blocking". This denotes the case where two copies of the same staple each bind to one of the two scaffold domains for that staple, thus preventing either copy from reaching its fully-bound state. An example configuration for such a state is shown in Fig. 5(e). These blocking states are long-lived on our simulation time scales and at $65^{\circ} \mathrm{C}$ we have never observed a blocked configuration involving two $\mathrm{R}$ staples to be resolved.

These blocking events will be most prevalent when the time scale for the intramolecular process of a staple binding to its second scaffold domain is of the same order as that for the intermolecular process of a staple initially binding to the scaffold. In the examples of blocking that we have observed, misbinding often plays a role in hindering the binding of a staple to its second scaffold domain. This could either be because the staple's second domain is itself involved in misbonding (for example, see the kymograph in Fig. 2 and the snapshots shown in Fig. 5, where a staple is correctly bound to scaffold domain 9 and misbonds to scaffold domain 8, eventually allowing a second staple to come into scaffold domain 10 and block the original staple) or because the second scaffold domain required for complete binding is partially blocked by misbonding by a different staple.

Recovering from blocking. Conceptually, the most straightforward way for the partial assembly to recover from blocking is by one of the blocking staples melting away from the scaffold. However, this is rare at $65^{\circ} \mathrm{C}$, because we have chosen to work in the "downhill" temperature regime, i.e. where the binding of every staple domain is downhill in free energy (as mentioned earlier, observing assembly in simulations at higher temperatures is less tractable because of the reduced binding rates and increased detachment rates). At higher temperatures, this mechanism is likely to be more important, for example where a staple is only stable when its second domain has bound. Blocking is likely to be less of a problem at these temperatures, and in particular at the temperature where a complete origami first becomes stable on annealing.

A potentially easier way to recover from blocking at $65^{\circ} \mathrm{C}$ is through one blocking staple displacing the other. The displacing staple cannot form a continuous helix when initiating this displacement. In this sense, it is a "remotetoehold" displacement. $\underline{50}$ The likelihood of this form of displacement will in part depend on any geometrical constraints stemming from the rest of the origami structure. For example, for blocks involving the M (or L) staples, if the adjacent $\mathrm{L}$ ( or $\mathrm{M}$ ) staple is correctly bound then the crossover will bring the unbound domains of the blocking staple strands in a favourable position to initiate displacement (e.g. Fig. 4 shows a fully bound L staple creating a more favourable geometry for the neighbouring $\mathrm{M}$ staple).

By contrast, for blocks involving $\mathrm{R}$ staples, the rest of the origami provides no such constraints, as in our small origami the $\mathrm{R}$ staples form single crossovers (Fig. 1). Instead the most likely configuration is one where there is coaxial stacking between the bound ends of the staples, so that the unbound domains are as far away as possible from each other. In this case, coaxial stacking refers to stacking between the adjacent ends of two staples. An example of this is seen for domains 9 and 10 in Fig. 5(f). Finally, misbonding of the blocking staples to other scaffold domains will again make displacement less likely.

The melting of one of the blocking strands can be assisted by misbonding from an another staple with a free domain. A possible example of this can be seen in Fig. 2, where the staple natively bonded to scaffold domain 9 competes for base pairs in scaffold domain 8 with a blocking staple from about $0.1 \mathrm{~s}$ to about $0.17 \mathrm{~s}$, after which the blocking staple falls off. This is illustrated with simulation snapshots in Fig. 5(c) and Fig. 5(d). By forming some misbonds with the scaffold domain, the misbonding strand can partially compensate for the lost base pairs on melting the blocking strand, in a process somewhat analogous to strand displacement. Although this kind of blocking resolution involves a reduction in base pairing, it is geometrically easier than displacement by the other blocking strand because the misbonding strand is adja- 
cent (along the scaffold strand) to the domain to which it misbonds.

The majority of the blocking that we observe involves the $\mathrm{R}$ staples, and at $65^{\circ} \mathrm{C}$ seems to be effectively irreversible. By contrast, blocking involving the $\mathrm{M}$ or $\mathrm{L}$ staples is more transient. Note that, while L and R staples can enhance M staple melting by misbonding, and M staples can do the same for L staples, $\mathrm{R}$ staples cannot be subject to any such effect. This is because the free ends belonging to the staple strands adjacent to the $\mathrm{R}$ staples are too far from the $\mathrm{R}$ staples' domains, a consequence of the single crossover that the $\mathrm{R}$ staple forms.

In fact, we have never seen blocking involving $\mathrm{M}(\mathrm{L})$ staples when the adjacent L (M) staple is fully bound. There are a number of factors that potentially contribute to this behaviour: (i) As discussed above, if a fully bound L (M) staple is already in place, an adjacent $\mathrm{M}(\mathrm{L})$ staple that subsequently binds its first domain will rapidly bind its second, leaving little opportunity for a blocking strand to bind. (ii) If a blocking pair of L (M) staples are in place, binding of the second domain of an adjacent $\mathrm{M}(\mathrm{L})$ staple may be hindered by misbonding from the free end of one of the blocking strands. (iii) In the above situation, the cost of forming the junction when binding the second domain will also be increased somewhat due to the presence of the singlestranded tails of the blocking strands. The tails may have to break stacking at the junction and will have reduced conformational entropy due to steric constraints caused by the presence of other nearby strands (a somewhat similar freeenergy penalty has been found for initiating displacement because the displacement generates a second single-stranded tail $\stackrel{43}{ }$ ). Interestingly, in separate simulations of an origami with a blocking pair of $\mathrm{L}(\mathrm{M})$ staples and an adjacent fully bound $\mathrm{M}(\mathrm{L})$ staple not only did we find that sometimes one of the blocking strands would displace the other, but that sometimes the fully bound staple would melt off.

Blocking in real origami self-assembly. Clearly, the relatively high concentrations that we are using play a role in the frequency of these blocking events, as they increase the rate of intermolecular binding without affecting intramolecular binding. However, although experimental origamis are typically assembled at much lower concentrations, there are strong reasons to suggest that blocking might still be a hindrance for assembly. Firstly, a full-size origami has many more staples, and a small number of key staples failing to fully bind due to blocking could be highly detrimental. Secondly, the longer scaffold strand and potentially multiple scaffold domains per staple strand will increase the time scale for intramolecular binding compared to our simple design, boosting the chance of staple blocks occurring. The longer scaffold strand leads to designs which often involve staples with domains that are much further apart along the scaffold strand than for our design, so that completing the binding of each staple can take much longer. Meanwhile, when staples that bind to three or more scaffold domains are used it is possible for the domains at the two ends of the staples to bind first, in which case the binding of the middle domain may be hindered by the absence of a free end to facilitate wrapping of the two strands around each other. 51 Thus it may be necessary for one of the end domains to dissociate before binding can be completed, a potentially slow process.

Effects analogous to staple blocking have been seen in experiments with DNA tweezers, with dimerisation between tweezers being observed. $\underline{52153}$ In addition, in the DNA walker experiments of Tomov et al., $\underline{54}$ increasing the fuel strand concentration was found to decrease the stepping yield for one of the designs, even though it increased the initial rate of stepping. Simultaneous binding of two copies of the fuel strand was identified as the cause of this reduction in yield. For that system, the effect was observed for fuel concentrations as low as $50 \mathrm{nM}$, while the distance between the two domains to which a single fuel strand was intended to bind was 5-10 nm. This observation suggests that staple blocking might be relevant at staple strand concentrations of $\sim 50 \mathrm{nM}$ and perhaps even lower, especially when remote domains of the scaffold must be bound by a single staple.

We note that, while the amount of time required might be prohibitively long in practice, 
displacement or melting of the blocking staples will eventually resolve the blocking we observe. However, one or more of the blocking staples may bind to another scaffold in the meantime, which would cause unwanted aggregates of multiple partially-formed origamis to form. The experiments of Bae et al., ${ }^{55}$ where origami assembly is initiated from a scaffold strand that is both under tension and fully loaded with staples that are only bound to a single domain, provide interesting insights into these processes. When the tension is reduced, assembly mediated by the displacement of blocking strands is observed, but the heterogeneity in the assembly products (as evidenced by the wide range of extensions observed when tension is applied to the product) indicates the possibility of persistent staple blocking. Note that the immobilization of the scaffold strand on a surface makes aggregation unlikely in this experiment.

Other simulation runs. We performed four simulation runs in total at this temperature $\left(65^{\circ} \mathrm{C}\right)$ - kymographs for the other runs are shown in Fig. S2, and show similar patterns of behaviour. In all four runs blocking prevents complete assembly, with the run described above getting closest to completion. At the end of the simulations, the other three runs had 10, 18 and 18 correctly bound domains, with 10, 4 and 4 domains blocked, respectively, compared to 20 correctly bound domains and 2 blocked domains for the simulation run described above. Longer simulations confirm that the blocking involving $\mathrm{R}$ strands is effectively irreversible on our time scales at this temperature.

Excess-staple assembly annealed at a higher temperature: $70^{\circ} \mathrm{C}$. We have seen that the assembly run with a large staple excess was hindered by staple blocking, preventing complete assembly on the time scales simulated. One way to resolve the blocks might be to increase the temperature somewhat, so that both partially-bound staples are more likely to melt off the scaffold during the simulation, and displacement is more likely due to enhanced fraying. We tested this idea by performing four runs beginning from the end points of previous lower temperature runs (which had been run for approximately $0.8 \mathrm{~s}$ ), and raising the temperature to $70^{\circ} \mathrm{C}$.

A kymograph of one of these runs is shown in Fig. 6. From this graph it is clear that, although the annealing is allowing blocks to be resolved, new blocks are also being formed, and so overall there is no net progress towards forming a complete origami. The same is true of the other three runs performed.

Resolution of blocking. Examples of the three possible ways of resolving blocking mentioned above are evident in the kymograph of Fig. 6, with snapshots illustrating these processes shown in Fig. 7 .

Firstly, we see an example of blocking resolution by displacement, in this case for an $R$ staple, in scaffold domains 21 and 22 at around $1.3 \mathrm{~s}$. The difficulty of this kind of displacement is highlighted in the simulation snapshots in Fig. 7(a), where the scaffold strand has both to break coaxial stacking and to bend quite sharply, a rare configuration, in order for the displacement to begin.

Secondly, we see an example of blocking resolution by melting for the $\mathrm{R}$ staple whose target is scaffold domains 15 and 16. At around $1.3 \mathrm{~s}$ the blocking staple bound to domain 15 melts off, and then approximately $0.05 \mathrm{~s}$ later the other staple binds to domain 15, completing its binding to the scaffold (Fig. 7(b)).

Thirdly, an example of misbonding aiding blocking resolution occurs for scaffold domain 8 , after slightly less than $1.2 \mathrm{~s}$. Here we see the loss of a blocking staple in scaffold domain 8 at the same time as the staple bound to scaffold domain 7 misbonds with domain 8 (Fig. 7(c)). It is likely that this misbonding plays a role in accelerating the melting of the blocking staple.

Formation of new blocks. However, at this higher temperature we also see instances of fully bound staples becoming partially unbound and then subsequently blocked. This behaviour is perhaps not surprising, as the higher temperature will slightly destabilise correctly bound staples as well as blocking ones.

An example of melting of a fully bound staple followed by blocking is given by the $\mathrm{R}$ staple bound to domains 3 and 4 . At around $2.12 \mathrm{~s}$ the part of the staple bound to domain 4 melts off, 


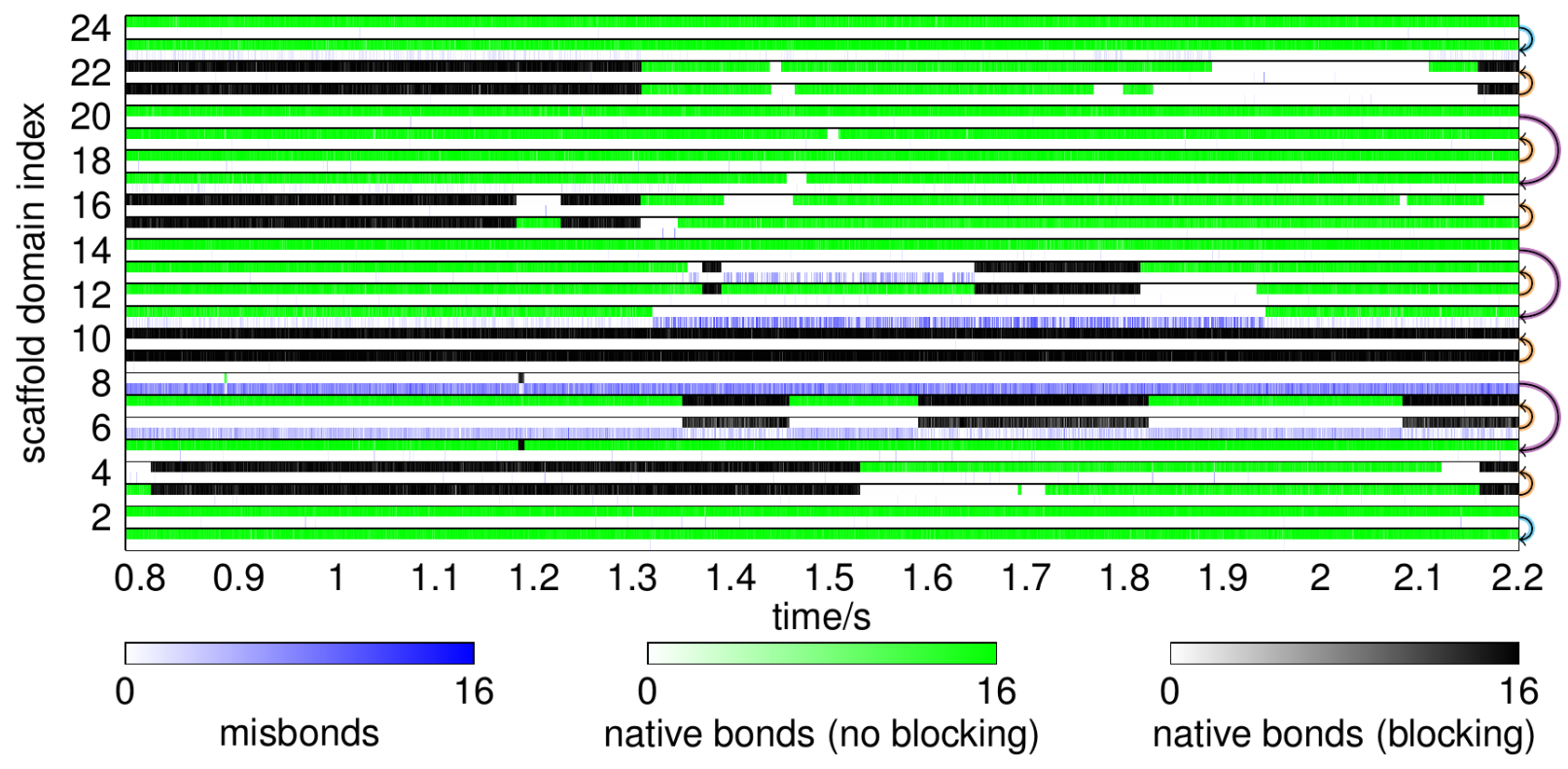

Figure 6: A kymograph for the annealing at $70^{\circ} \mathrm{C}$ of an assembly produced by a $0.8 \mathrm{~s}$ simulation at $65{ }^{\circ} \mathrm{C}$ with a large staple excess.

and shortly after that a copy of the staple comes in and binds to domain 4, forming a block.

At the start of the simulation an L staple is correctly bound to scaffold domains 12 and 13 . However at around $1.3 \mathrm{~s}$ domain 13 melts, probably facilitated by misbonding from the staple bound to domain 14. This allows another copy of the original staple to bind to domain 13, thus generating a blocked configuration.

Overall, our annealing simulations at $70^{\circ} \mathrm{C}$ with an excess of staples showed no progress, that is, on average there are no fewer blocking staples at the end of the simulation than there were at the beginning. In other words, the enhanced rate of resolving blocking appears to be roughly equal to the increased rate of forming new blocking pairs. If this really is the case, it would imply that the thermodynamically favourable state for the system is a partially assembled origami with some blocking strands. This may be partly due to the small simulation box and large staple excess (equivalently, very high strand concentration). The scaffold strand gains configurational entropy when the origami has blocks rather than being correctly bound, as an origami with blocks has fewer junctions constraining the scaffold strand. However, the system also loses translational entropy (equivalent to the translational entropy one of the staples would have had if it could diffuse freely) for each blocking pair. By raising the strand concentration, we reduce this loss of translational entropy (without significantly affecting the configurational entropy of the scaffold strand), shifting the balance in favour of staple blocking.

Assembly with a stoichiometric strand mixture. Having established that, for our high-concentration, large-staple-excess simulations, staple strand blocking prevents completed assembly, at least on the time scales accessible to us through simulation, the obvious question is how we can we get around this problem. We have seen above that simply annealing such assemblies at higher temperature is not enough.

One approach would be to lower the staple concentration to something comparable to the concentrations used in experiment. This would have two positive effects: (i) it would increase the translational entropy penalty for blocking, and (ii) it would lead to a greater separation between the time scales for a staple binding its second domain and the initial binding of a staple to the scaffold, thus making it more likely that each staple would fully bind before it could be blocked by a second staple. Unfortunately it would no longer be feasible to observe assem- 
(a)

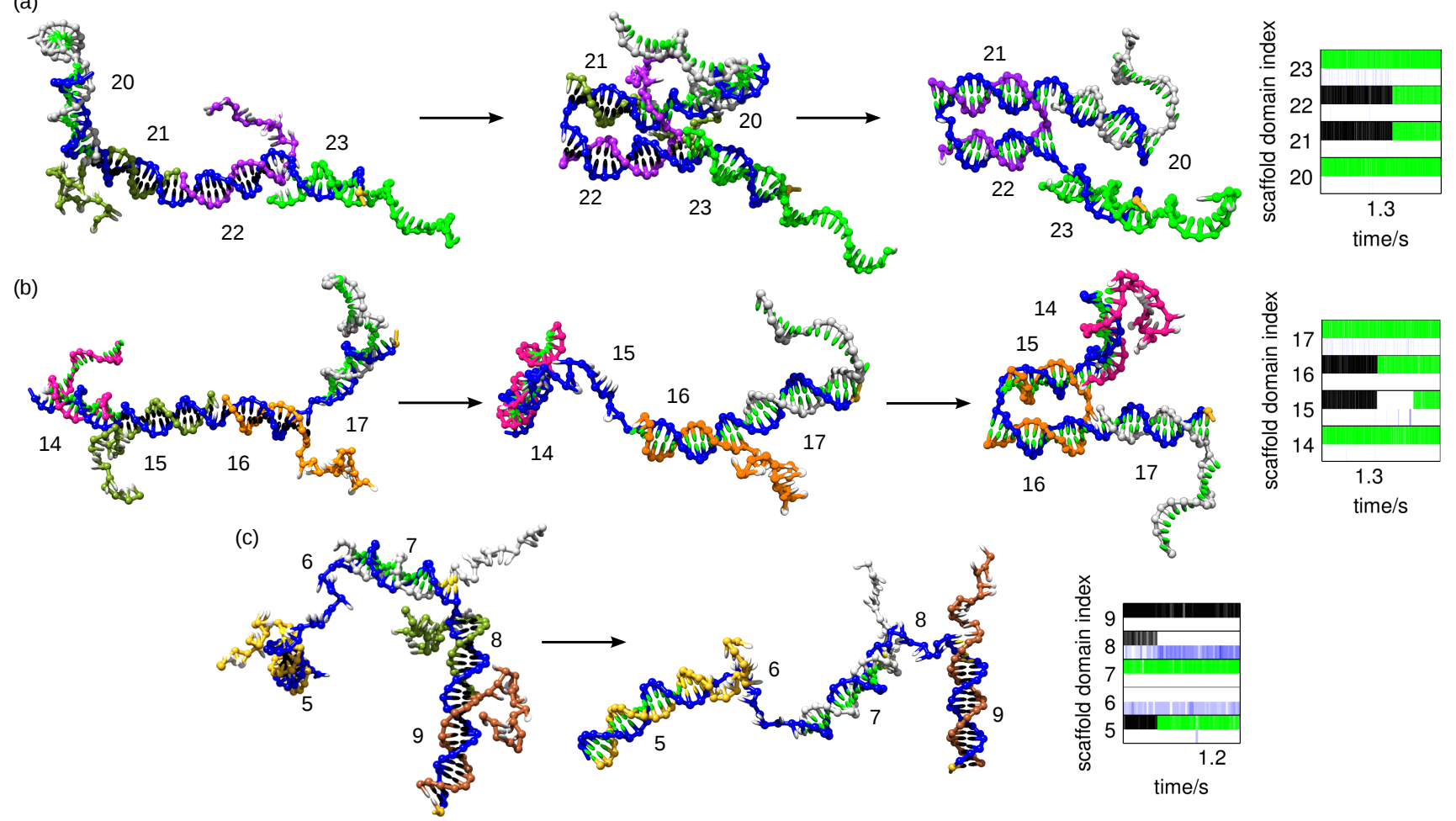

Figure 7: Simulation snapshots and corresponding kymographs (right) showing resolution of blocking by various mechanisms during annealing at $70^{\circ} \mathrm{C}$ with a large staple excess. For clarity, only the relevant section of the scaffold is shown in each case. Note that some staple domains are bound to a scaffold domain that is not shown. The nucleotides are coloured as in Fig. 3 and Fig. 5 . (a) Blocking resolution by displacement. The purple strand bound to domain 22 displaces its competing blocking strand bound to domain 21, thus achieving complete binding. The middle panel shows an intermediate in the strand-displacement reaction. (b) Blocking resolution by melting. The olive blocking strand bound to domain 15 melts off, making this domain available for binding. About $0.05 \mathrm{~s}$ later, the orange staple binds to domain 15, completing its binding to the scaffold. (c) Blocking resolution facilitated by misbonding. In the left panel, the olive strand (bound to domain 8 ) and golden strand (bound to domain 5) are blocking each other. The gray strand (natively bound to domain 7) is misbonding with scaffold domain 8, preventing the olive strand from fully binding to that domain. In the right panel, the olive strand has melted off, having been unable to fully bind to domain 8 . The golden strand is now no longer blocked and is free to bind to domain 8 . Note that the brown strand (bound to domain 9) is also misbonding with the now vacated scaffold domain 8 . 
bly in our simulations under these conditions, as the vast majority of the computational effort would then be spent simulating strands diffusing around the box.

We take an alternative approach: while keeping the same simulation box size, we simulate a stoichiometric assembly, with only one copy of each staple in the simulation box. The design of the origami remains unchanged. Four simulations were run for each of the five temperatures considered. Fig. 8 shows example kymographs for simulations at $50^{\circ} \mathrm{C}, 60^{\circ} \mathrm{C}$ and $65^{\circ} \mathrm{C}$ : the simulation represented in Fig. $8 \mathrm{~b}$ is the only one of the twenty runs that led to complete assembly of the origami.

General features. The features of the stoichiometric assembly are in some ways very similar to those of the excess-staple assembly. The staples can bind more or less in parallel, misbonding is still evident, and the time scales for intra- and inter-molecular binding are still comparable, even though binding the first staple domain is now significantly slower due to the lower staple concentration (fortunately the lower number of nucleotides in the simulation box means that each simulation step is cheaper, so that simulating the assembly is still feasible). As with the excess-staple assembly, binding the second staple domain can be slow, especially at $50{ }^{\circ} \mathrm{C}$ where there is a lot of misbonding, and in the absence of staples bound to nearby scaffold domains which might help to provide a favourable scaffold geometry (cooperativity in forming the fourth domain around a four-way junction with two crossovers is still very clear).

However, by design the most detrimental effect for our assembly simulations, staple blocking, is no longer possible. As a result, a complete assembly was achieved, at $60{ }^{\circ} \mathrm{C}$. The kymograph for this simulation is shown in Fig. 8(b) and snapshots illustrating the pathway are shown in Fig. 9 (note that the complete origami (Fig. 9(i)) invariably has a nonplanar configuration). In addition, in Fig. S4, we report how a number of geometric descriptors (such as the radius of gyration of the scaffold) evolve with time for this trajectory. Note that this trajectory was the only one to reach completion of the four simulations run at $60^{\circ} \mathrm{C}$, all of which ran for roughly the same length of time $(2.8 \mathrm{~s})$. However, it seems very likely that the others would have also completed their assembly relatively easily if given more time as there are no obvious kinetic traps.

Optimal assembly window. As expected, we find that there is an optimal temperature window for observing assembly on our simulation time scales. Misbonding is observed more frequently at lower temperatures, as can clearly be seen from the regions of blue in Fig. 8 (a). The misbonding hinders the assembly, because it makes it harder for both the correct staple to bind and for the staple involved in misbonding to bind to its correct domains. On the other hand, at higher temperatures (see e.g. Fig. 8(c) and Fig. S3(b)) the staple binding rate is reduced and the rate of melting correctly bound staples increases. These competing effects lead to assembly being fastest at intermediate temperatures. For the stoichiometric mixture, we ran simulations at $50^{\circ} \mathrm{C}, 55^{\circ} \mathrm{C}, 60^{\circ} \mathrm{C}, 65^{\circ} \mathrm{C}$ and $70^{\circ} \mathrm{C}$. We found that not only was the only complete assembly observed at $60^{\circ} \mathrm{C}$, but on average simulations at this temperature showed the largest number of correctly bound domains for any given simulation time.

\section{Conclusions}

This work represents a significant milestone in the simulation of DNA nanotechnology: we have simulated the complete assembly of by far the largest DNA nanostructure ever studied using a nucleotide-level, continuous-space model. In the course of our investigations we have identified a number of interesting features of origami self-assembly.

We have identified staple blocking as the biggest barrier to assembly for our system. It is important to note that our assembly is atypical in two ways: the strand concentration is roughly $1000 \times$ larger than for a typical assembly, and the origami structure is roughly $20 \times$ smaller than a typical origami. The very high concentration will make blocking more likely, as it increases the binding rate of each staple's initial domain without affecting the binding rate 
(a)

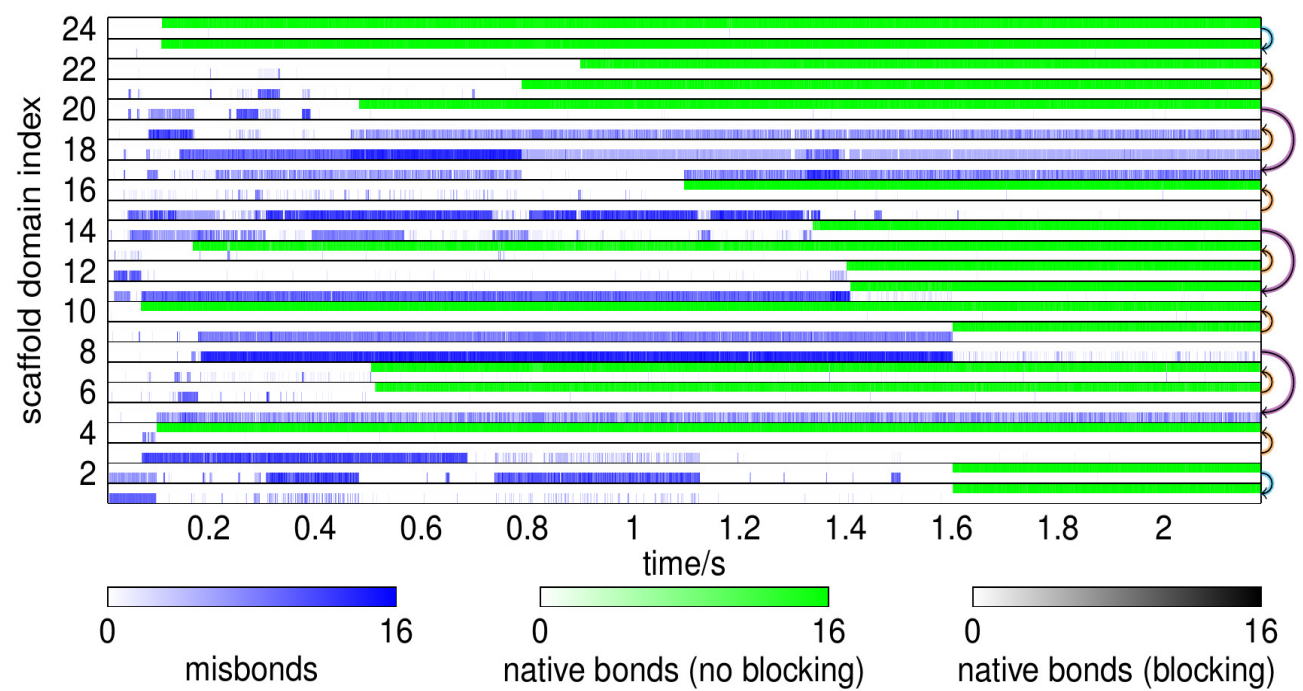

(b)

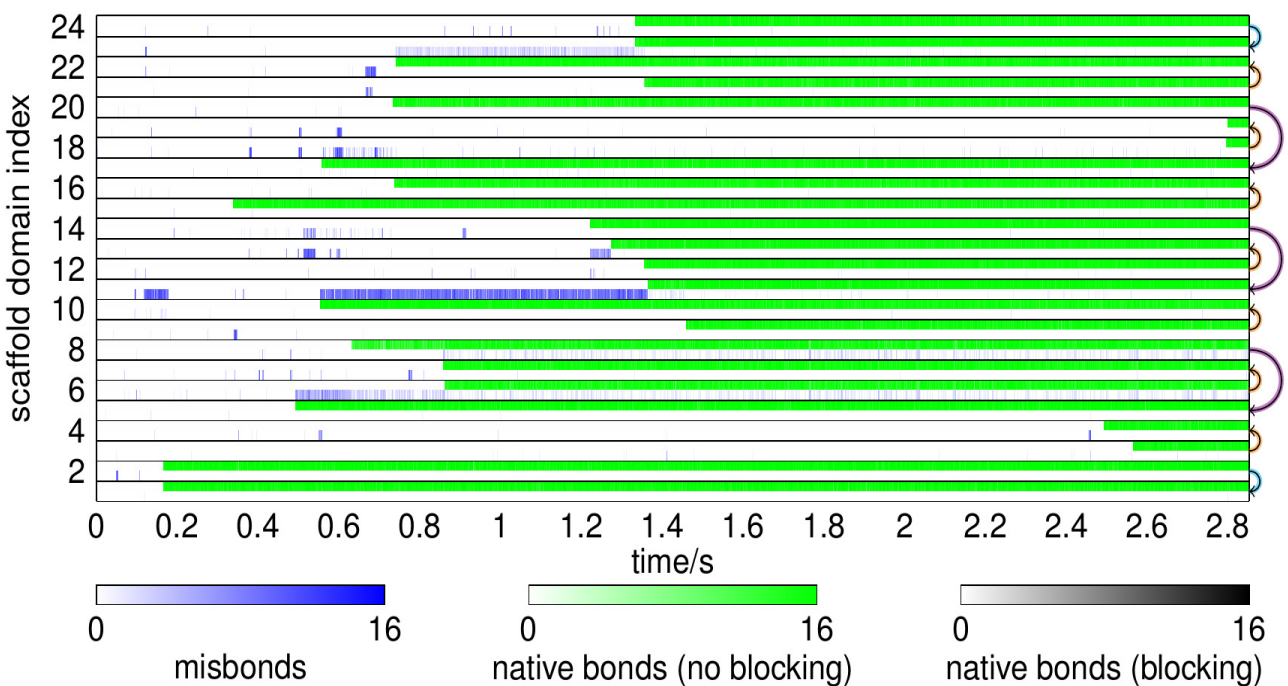

(c)

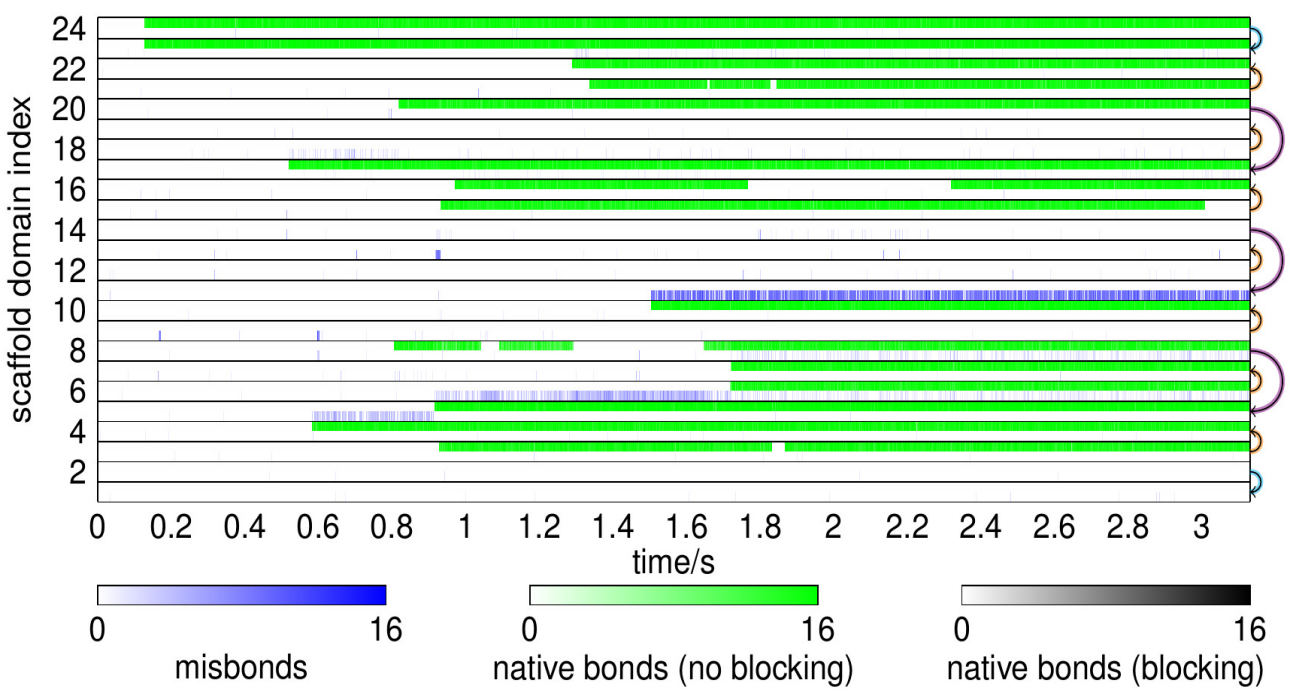

Figure 8: Kymographs for stoichiometric assembly simulations at (a) $50{ }^{\circ} \mathrm{C}$, (b) $60{ }^{\circ} \mathrm{C}$ and (c) $65{ }^{\circ} \mathrm{C}$. 


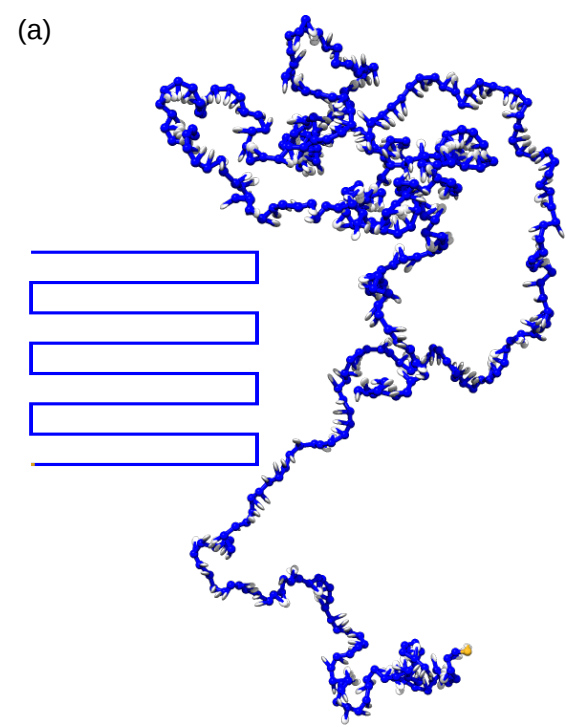

(d)

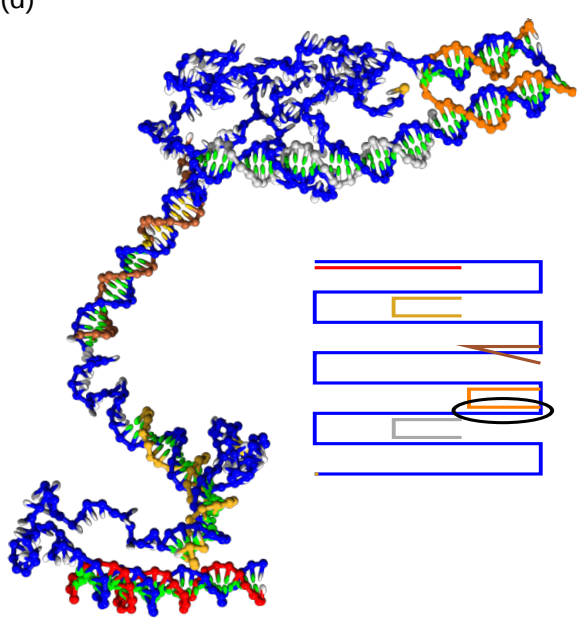

(g)

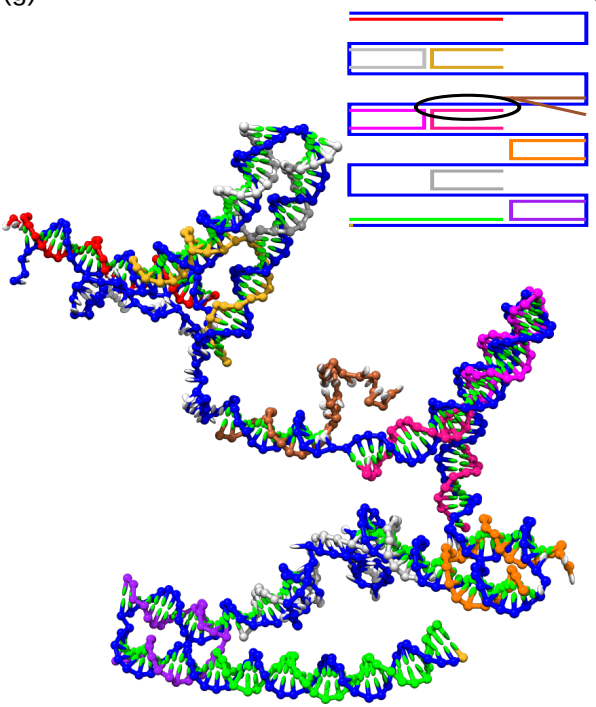

(b)

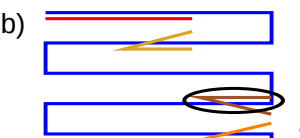

(b)

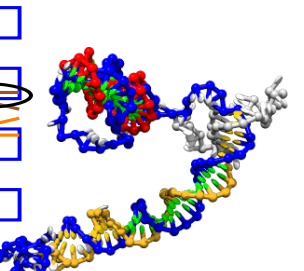

(c)

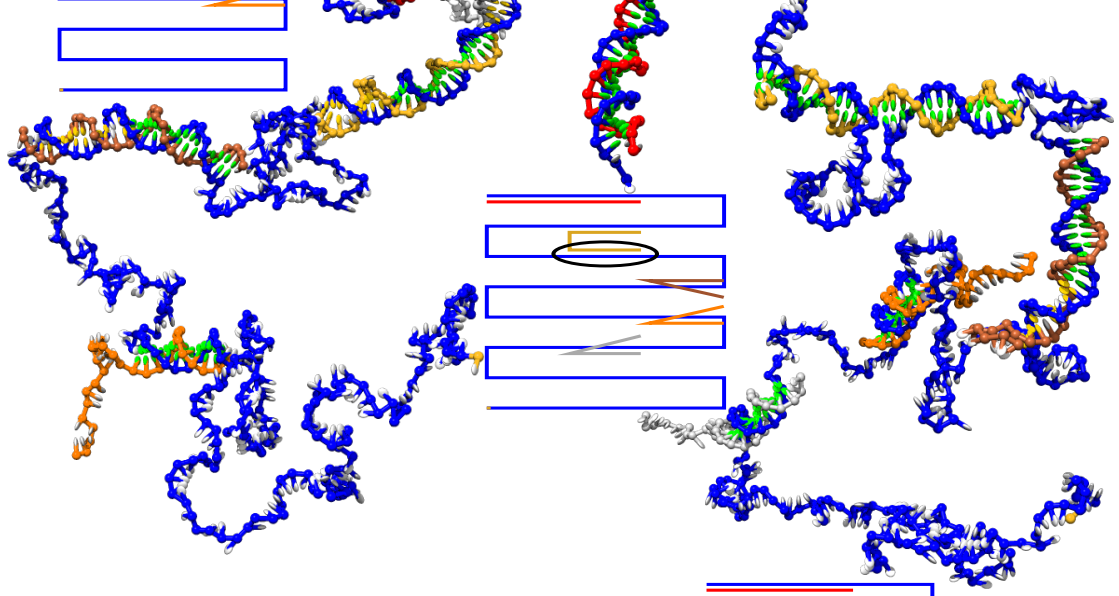

(e)

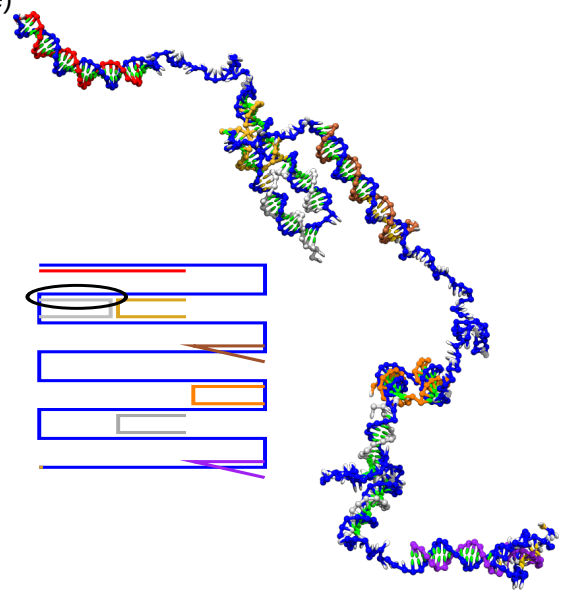

(f)

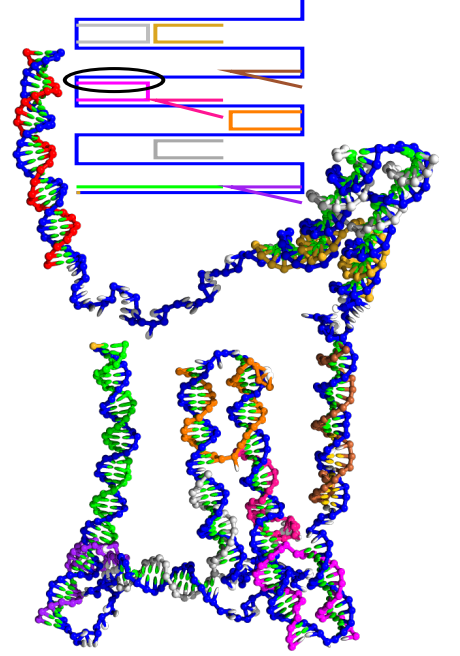

(h)

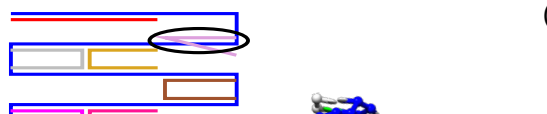

(i)
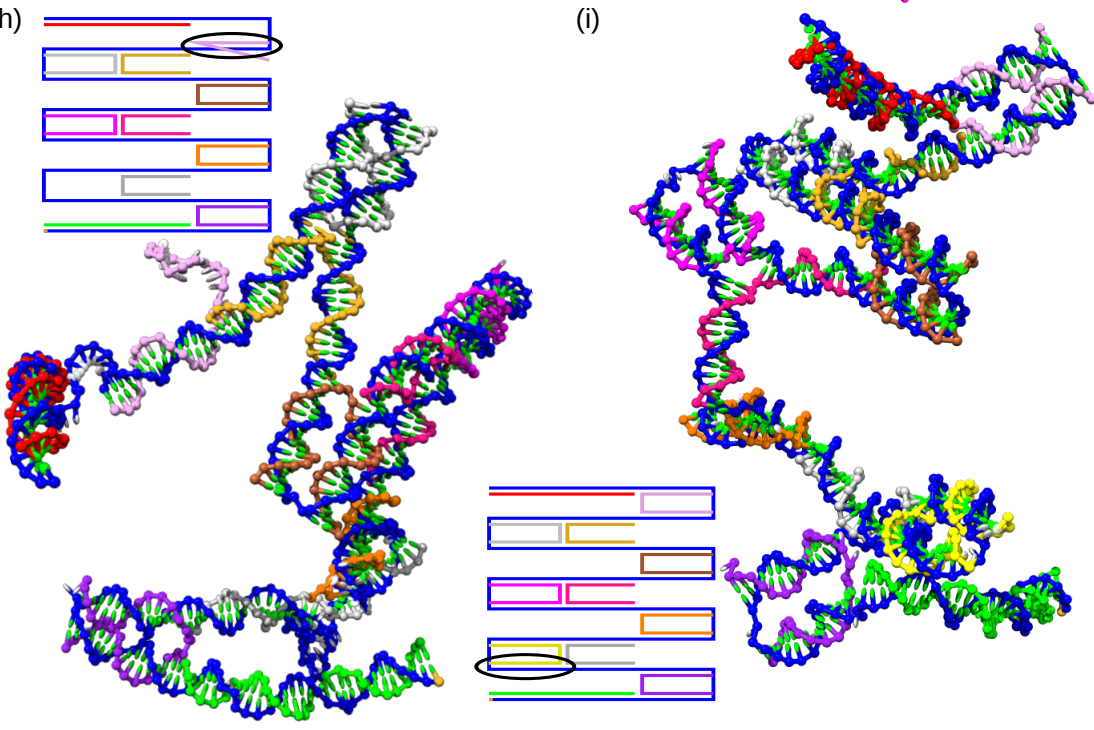

Figure 9: Snapshots along the pathway to complete assembly for the trajectory of Fig. 8(b). The completely assembled origami is shown in (i) - note that due to the small number of crossovers the structure is not very well constrained and so looks quite disordered compared to the configuration shown in Fig. 1, where the origami is constrained to be planar by adsorption onto a surface. The scaffold strand is shown in blue and staple strands are shown in other colours. Unpaired base sites are white, natively-bonded base sites are green, and misbonded base sites are yellow. Only staple strands with at least one base pair formed with the scaffold are shown. Cadnano ${ }^{56}$-style schematics are shown, illustrating the assembly progress, with ovals highlighting the most recently bound scaffold domain. 
for its second domain. Additionally, in order to see assembly on reasonable computational time scales, we had to work at a temperature where the binding of any staple to any scaffold domain is free-energetically downhill, thus helping to stabilize the blocked state.

Nevertheless, there are several factors which suggest that staple blocking might also be important for experimental origami self-assembly. They are all related to the fact that experimental origamis are typically much larger and more complex than the simple design we have simulated. Firstly, in our design, a partially bound staple must only bind to one more scaffold domain, and that domain will not be too far away from the first, so that it is not too difficult for a staple to complete its binding. On the other hand a typical origami design, which is much larger and more complicated, will imply a much slower binding completion rate for each staple, leaving a much larger window of opportunity for a competing staple to bind to the scaffold in the mean time. In addition, a typical origami will need to incorporate many more staple strands, so that even a low probability of a given staple becoming blocked could lead to the blocking of a significant number of staples. Finally, staples which bind particularly distant stretches of the scaffold together are the most likely to be hindered by staple blocking, and are also likely to be the most vital for the proper folding of the origami.

Therefore, it seems reasonable to conclude that staple blocking may be an important obstacle to assembly for some designs, and certainly for unusually high strand concentrations. As noted earlier, experimental results for a DNA walker $\underline{54}$ show an effect analogous to blocking occurring at strand concentrations as low as $50 \mathrm{nM}$.

We emphasise that, whereas in our simulations a partially formed origami with a few staple blocks appeared to be the thermodynamic equilibrium near to the melting point, this will almost certainly not be the case at the low strand concentrations typical of experiment ( 10 nM). In a low-concentration assembly, any blocking staples that bind will be slowly replaced by correct binding, as long as the as- sembly is cooled sufficiently slowly to allow this to happen. We can see this from the very large free-energy penalty suffered from a blocking pair of staples (compared to a single fully bound staple), due to the loss of translational entropy of one of the staples. Thus it should be possible to overcome staple blocking with sufficiently slow annealing. However, if the system is cooled too quickly, or deliberately quenched to a lower temperature as in Ref. 27, blocking is one of the likely mechanisms preventing robust assembly of the target structure, and may lead to aggregation.

Ke and coworkers ${ }^{29}$ found that positioning staple breaks so that each staple had a long (14base-pair) middle domain and many shorter domains permitted high yields, while positioning the longest domains at the ends of the strands or having equal lengths for all domains led to little or no successful assembly. It is interesting to note that the success of the high-yield strategy is consistent with our findings regarding staple blocking. For an annealed assembly with staples with long middle domains (the highyield strategy), we would expect each staple to experience the following (and therefore avoid being blocked): (i) initially, in a certain temperature window one copy of the staple is able to bind its long domain, leaving only shorter domains available for competing copies, so that any competing copies which happen to bind will quickly melt off (ii) either at that temperature or as the temperature is lowered further, this partially bound staple will be able to bind to the other, shorter scaffold domains before any copies can come in and block it.

In general the assembly we have studied is relatively parallel in nature - that is, assembly in distant parts of the structure occurs relatively independently, with no set order in which the staples bind. This is again probably in part due to the necessity of simulating the assembly at a temperature where binding of a staple to any scaffold domain is stable, in order to make assembly computationally tractable. This parallel aspect would also be expected to apply to larger and more complex origamis when quenched to this temperature regime. However, our results also show evidence for cooperativ- 
ity between staples in certain cases, which occurs when staples bring together distant parts of the scaffold strand, enhancing the geometry for other staples to bind their second domains. This inter-staple cooperativity, which has been incorporated in theoretical studies of origami assembly, $\stackrel{31}{13}$ is particularly evident in the case of the four-way junction formed by the $\mathrm{L}$ and $\mathrm{M}$ staples.

For a typical experimental design, which will be larger and potentially more complex than our simple origami, there is a greater potential for cooperativity, as (i) a partially bound staple can have more neighbouring staples than for our design, which can lead to an even more constrained, and so more favourable, local scaffold geometry, (ii) scaffold domains which are to be connected by a single staple may be separated by a much greater distance along the scaffold strand than for our small origami design, so that larger gains are made by constraining the scaffold geometry, and (iii) on annealing in experiment assembly is likely to first occur when there is a nucleation barrier to assembly, and where it is more favourable to first bind staples that involve less loss in scaffold entropy. This enhanced cooperativity suggests the possibility of there being nucleation sites in partially assembled origamis, where closing one staple accelerates the closing of the neighbouring staple, which on closing aids its neighbour, and so on. Having said this, it is also likely that the self-assembly of such large structures would also have a parallel aspect.

In addition, we expect a clear intra-staple cooperativity effect at experimental strand concentrations. Although in our simulations the time scales for binding the first staple domain are similar to those of binding the second staple domain, this will not be the case at experimental concentrations, which will greatly reduce the rate of binding the first staple domain while leaving the rate of binding the second domain unchanged. Thus, once a staple has bound its first domain to the scaffold, the second domain will bind relatively very fast for the origami we have considered.

Optimal temperature windows for origami assembly have been identified in experiment. 27
We see similar features in our simulations. At lower temperatures, misbonding is more prevalent, which hinders correct binding. For the more complex designs typical of experiment, it is likely that this effect will be even more pronounced, as there is more opportunity for misbonding to cause kinetic traps if the assembly is cooled too rapidly. Meanwhile, at high temperatures staples form native base pairs with the scaffold more slowly, and staples that have partially or fully bound to the scaffold are more likely to melt off.

However, in our simulations, in order to see assembly on computationally feasible time scales, the temperature had to be sufficiently low that the native binding of a staple to a scaffold domain was always free-energetically downhill. This condition, though, leads to, for example, blocking being a potential kinetic trap hindering assembly. In experiment, it is likely that assembly occurs with highest fidelity at somewhat higher temperatures where there is a nucleation free-energy barrier to assembly that helps prevent the formation of incorrect structures. Performing assembly simulations in this regime, which would require the use of advanced rare-event techniques, is a challenge we hope to address in future work.

The work described here is very much complementary to domain-level kinetic models of origami assembly. 30131 Here, we are able to provide detailed microscopic insights and naturally capture the geometric and topological properties of the strands involved, but are much more restricted in time and length scales than the kinetic models. Furthermore, oxDNA can help parameterize the polymer modelling used in such domain-level models through detailed characterization of the thermodynamics of smaller model motifs.

\section{Methods}

All of the assembly simulations were run with a Brownian dynamics simulation algorithm, consisting of a molecular dynamics (MD) algorithm plus an Andersen-like thermostat. The simulation parameters used are given in Table S1. 
The initial configurations for the assembly runs were generated by simulating the appropriate single strands in a periodic box (with artificial polyA sequences to prevent any base pairing) for $10^{7}$ simulation steps. The assembly simulations were run for up to $6 \times 10^{10}$ simulation steps. In order to make simulating thousands of nucleotides with oxDNA feasible, the algorithm was run on graphical processing units (GPUs), devices suitable for parallel computation of pairwise forces, giving up to a $25 \times$ speed-up over a typical CPU.

Inferring the physical time elapsed during an assembly run simulated with an Andersen-like thermostat is not straightforward. $\stackrel{37}{ }$ The energy and length scales within the model suggest a conversion from simulation time units to physical units of $3.03 \times 10^{-12}$ s per simulation time unit. However, this is likely to lead to an underestimate of the times relevant to self-assembly because it does not take into account the reduction in time scale separations often inherent in coarse graining, e.g. speeding up diffusion relative to microscopic time scales. ${ }^{[57}$ In fact, the appropriate time unit conversion for the model will likely be different for different processes. Here, we use a conversion factor obtained by considering the rate of hybridization of a 16base-pair domain, as this is likely to be the important time scale for the assembly. This leads us to a rough conversion factor of $10^{-8} \mathrm{~s}$ per simulation time unit or $5 \times 10^{-11}$ s per simulation step (the details of the calculation that led to this result are given in the Supporting Information).

\section{Acknowledgements}

The authors are grateful for financial support from the EPSRC. The work presented here made use of the Emerald High Performance Computing facility made available by the Centre for Innovation, and the University of Oxford Advanced Research Computing (ARC) facility (http://dx.doi.org/10.5281/zenodo.22558). LR acknowledges support from the Austrian Research Fund (FWF) through the Lise-Meitner Fellowship M 1650-N27.
Supporting Information Available: Details of the simulation parameters used for this work; details of the procedure to determine the estimated time unit conversion; the DNA sequences used for the simulations described in this work; snapshots from the beginning of the assembly simulations; additional kymographs for the $65^{\circ} \mathrm{C}$ excess-staple assembly simulations and stoichiometric assembly simulations. This material is available free of charge via the Internet at http://pubs.acs.org.

Data access: The data used to generate the kymographs in this work is available for download from the Oxford University Research Archive with DOI 10.5287/bodleian:dr26xx669. The simulation package used to generate all the results described in this work is publicly available and can be found at http://dna.physics.ox.ac.uk.

\section{References}

1. Shih, W. M.; Lin, C. Knitting Complex Weaves with DNA Origami. Curr. Op. Struct. Biol. 2010, 20, 276-282.

2. Pinheiro, A. V.; Han, D.; Shih, W. M.; Yan, H. Challenges and Opportunities for Structural DNA Nanotechnology. Nat. Nano 2011, 6, 763-772.

3. Tørring, T.; Voigt, N. V.; Nangreave, J.; Yan, H.; Gothelf, DNA Origami: a Quantum Leap for Self-Assembly of Complex Structures. Chem. Soc. Rev. 2011, 40, 5636-5646.

4. Endo, M.; Yang, Y.; Sugiyama, H. DNA Origami Technology for Biomaterials Applications. Biomater. Sci. 2013, 1, 347360 .

5. Linko, V.; Dietz, H. The Enabled State of DNA Nanotechnology. Curr. Opin. Biotech. 2013, 24, 555-561.

6. Rothemund, P. W. K. Folding DNA to create nanoscale shapes and patterns. Nature 2006, 440, 297-302. 
7. Douglas, S. M.; Dietz, H.; Liedl, T.; Högberg, B.; Graf, F.; Shih, W. M. SelfAssembly of DNA into Nanoscale ThreeDimensional Shapes. Nature 2009, 459, 414-418.

8. Dietz, H.; Douglas, S. M.; Shih, W. M. Folding DNA into Twisted and Curved Nanoscale Shapes. Science 2009, 325, 725730

9. Han, D.; Pal, S.; Nangreave, J.; Deng, Z.; Liu, Y.; Yan, H. DNA Origami with Complex Curvatures in Three-Dimensional Space. Science 2011, 332, 342-346.

10. Liedl, T.; Högberg, B.; Tytell, J.; Ingbe, D. E.; Shih, W. M. Self-Assembly of Three-Dimensional Prestressed Tensegrity Structures from DNA. Nature Nanotech. 2010, 5, 520-524.

11. Rajendran, A.; Endo, M.; Sugiyama, H. Single-Molecule Analysis Using DNA Origami. Angew. Chem. Int. Edit. 2012, $51,874-890$.

12. Tsukanov, R.; Tomov, T. E.; Masoud, R.; Drory, H.; Plavner, N.; Liber, M.; Nir, E. Detailed Study of DNA Hairpin Dynamics Using Single-Molecule Fluorescence Assisted by DNA Origami. J. Phys. Chem. B 2013, 117, 11932-11942.

13. Wickham, S. F. J.; Endo, M.; Katsuda, Y.; Hidaka, K.; Bath, J.; Sugiyama, H.; Turberfield, A. J. Direct Observation of Stepwise Movement of a Synthetic Molecular Transporter. Nature Nanotech. 2011, $6,166-169$.

14. Jiang, Q.; Song, C.; Nangreave, J.; Liu, X.; Lin, L.; Qiu, D.; Wang, Z.-G.; Zou, G.; Liang, X.; Yan, H. et al. DNA Origami as a Carrier for Circumvention of Drug Resistance. J. Am. Chem. Soc. 2012, 134, 13396-13403.

15. Douglas, S. M.; Bachelet, I.; Church, G. M. A Logic-Gated Nanorobot for Targeted Transport of Molecular Payloads. Science 2012, 335, 831-834.
16. Langecker, M.; Arnaut, V.; Martin, T. G.; List, J.; Renner, S.; Mayer, M.; Dietz, H.; Simmel, F. C. Synthetic Lipid Membrane Channels Formed by Designed DNA Nanostructures. Science 2012, 338, 932-936.

17. Burns, J. R.; Gpfrich, K.; Wood, J. W.; Thacker, V. V.; Stulz, E.; Keyser, U. F.; Howorka, S. Lipid-Bilayer-Spanning DNA Nanopores with a Bifunctional Porphyrin Anchor. Angew. Chem. Int. Edit. 2013, 52, 12069-12072.

18. Bell, N. A. W.; Engst, C. R.; Ablay, M.; Divitini, G.; Ducati, C.; Liedl, T.; Keyser, U. F. DNA Origami Nanopores. Nano Lett. 2012, 12, 512-517.

19. Pfitzner, E.; Wachauf, C.; Kilchherr, F.; Pelz, B.; Shih, W. M.; Rief, M.; Dietz, H. Rigid DNA Beams for High-Resolution Single-Molecule Mechanics. Angew. Chem. Int. Edit. 2013, 52, 7766-7771.

20. Bai, X.-C.; Martin, T. G.; Scheres, S. H. W.; Dietz, H. Cryo-EM Structure of a 3D DNA-Origami Object. Proc. Nat. Acad. Sci. USA 2012, 109, 20012-20017.

21. Yoo, J.; Aksimentiev, A. In Situ Structure and Dynamics of DNA Origami Determined through Molecular Dynamics Simulations. Proc. Natl. Acad. Sci. USA 2013, 110, 20099-20104.

22. Castro, C. E.; Kilchherr, F.; Kim, D.-N.; Shiao, E. L.; Wauer, T.; Wortmann, P.; Bathe, M.; Dietz, H. A Primer to Scaffolded DNA Origami. Nat. Methods 2011, 8, 221229 .

23. Kim, D.-N.; Kilchherr, F.; Dietz, H.; Bathe, M. Quantitative prediction of 3D Solution Shape and Flexibility of Nucleic Acid Nanostructures. Nucleic Acids Res. 2012, 40, 2862-2868.

24. Pan, K.; Kim, D.-N.; Zhang, F.; Adendorff, M. R.; Yan, H.; Bathe, M. LatticeFree Prediction of Three-Dimensional Structure of Programmed DNA Assemblies. Nat. Comm. 2014, 5, 5578. 
25. Arbona, J. M.; Aimé, J.-P.; Elezgaray, J. Modeling the Mechanical Properties of DNA Nanostructures. Phys. Rev. E 2012, 86, 051912.

26. Wei, X.; Nangreave, J.; Liu, Y. Uncovering the Self-Assembly of DNA Nanostructures by Thermodynamics and Kinetics. Acc. Chem. Res. 2014, 47, 1861-1870.

27. Sobczak, J.-P. J.; Martin, T. G.; Gerling, T.; Dietz, H. Rapid Folding of DNA into Nanoscale Shapes at Constant Temperature. Science 2012, 338, 1458-1461.

28. Wagenbauer, K. F.; Wachauf, C. H.; Dietz, H. Quantifying Quality in DNA SelfAssembly. Nat. Comm. 2014, 5, 3691.

29. Ke, Y.; Bellot, G.; Voigt, N. V.; Fradkov, E.; Shih, W. M. Two Design Strategies for Enhancement of MultilayerDNAOrigami Folding: Underwinding for Specific Intercalator Rescue and Staple-Break Positioning. Chem. Sci. 2012, 3, 25872597.

30. Dunn, K. E.; Dannenberg, F.; Ouldridge, T. E.; Kwiatkowska, M.; Turberfield, A. J.; Bath, J. Guiding the Folding Pathway of DNA Origami. Nature 2015, 525, 82-86.

31. Dannenberg, F.; Dunn, K. E.; Bath, J.; Kwiatkowska, M.; Turberfield, A. J.; Ouldridge, T. E. Modelling DNA Origami Self-Assembly at the Domain Level. arXiv:1509.03066 2015,

32. Arbona, J. M.; Elezgaray, J.; Aimé, J.-P. Modelling the Folding of DNA Origami. EPL 2012, 100, 28006.

33. Cademartiri, L.; Bishop, K. J. M. Programmable Self-Assembly. Nature Materials 2015, 14, 2-9.

34. Ke, Y.; Ong, L. L.; Shih, W. M.; Yin, P. Three-Dimensional Structures SelfAssembled from DNA Bricks. Science 2012, 338, 1177-1183.
35. Maffeo, C.; Yoo, J.; Comer, J.; Wells, D. B.; Luan, B.; Aksimentiev, A. Close Encounters with DNA. Journal of Physics: Condensed Matter 2014, 26, 413101.

36. Ouldridge, T. E.; Louis, A. A.; Doye, J. P. K. Structural, Mechanical and Thermodynamic Properties of a Coarse-Grained Model of DNA. J. Chem. Phys. 2011, 134, 085101.

37. Doye, J. P. K.; Ouldridge, T. E.; Louis, A. A.; Romano, F.; Šulc, P.; Matek, C.; Snodin, B. E. K.; Rovigatti, L.; Schreck, J. S.; Harrison, R. M. et al. Coarse-Graining DNA for Simulations of DNA Nanotechnology. Phys. Chem. Chem. Phys. 2013, 15, 20395-20414.

38. Ouldridge, T. E.; Hoare, R. L.; Louis, A. A.; Doye, J. P. K.; Bath, J.; Turberfield, A. J. Optimizing DNA Nanotechnology through Coarse-Grained Modeling: A Two-Footed DNA Walker. ACS Nano 2013, 7, 2479-2490.

39. Šulc, P.; Ouldridge, T. E.; Romano, F.; Doye, J. P. K.; Louis, A. A. Simulating a Burnt-Bridges DNA Motor with a CoarseGrained DNA Model. Nat. Comput. 2014, 13, 535-547.

40. Ouldridge, T. E.; Louis, A. A.; Doye, J. P. K. DNA Nanotweezers Studied with a Coarse-Grained Model of DNA. Phys. Rev. Lett. 2010, 104, 178101.

41. Ouldridge, T. E.; Šulc, P.; Romano, F.; Doye, J. P. K.; Louis, A. A. DNA Hybridization Kinetics: Zippering, Internal Displacement and Sequence Dependence. Nucleic Acids Res. 2013, 41, 8886-8895.

42. Machinek, R. R. F.; Ouldridge, T. E.; Haley, N. E. C.; Bath, J.; Turberfield, A. J. Programmable Energy Landscapes for Kinetic Control of DNA Strand Displacement. Nat. Comm. 2014, 5, 5324.

43. Srinivas, N.; Ouldridge, T. E.; Šulc, P.; Schaeffer, J. M.; Yurke, B.; Louis, A. A.; 
Doye, J. P. K.; Winfree, E. On the Biophysics and Kinetics of Toehold-Mediated DNA Strand Displacement. Nucleic Acids Res. 2013, 41, 10641-10658.

44. Rovigatti, L.; Šulc, P.; Reguly, I. Z.; Romano, F. A Comparison Between Parallelization Approaches in Molecular Dynamics Simulations on GPUs. J. Comput. Chem. 2015, 36, 1-8.

45. Šulc, P.; Romano, F.; Ouldridge, T. E.; Rovigatti, L.; Doye, J. P. K.; Louis, A. A. Sequence-Dependent Thermodynamics of a Coarse-Grained DNA Model. J. Chem. Phys. 2012, 13\%, 135101.

46. Snodin, B. E. K.; Randisi, F.; Mosayebi, M.; Šulc, P.; Schreck, J. S.; Romano, F.; Ouldridge, T. E.; Tsukanov, R.; Nir, E.; Louis, A. A. et al. Introducing Improved Structural Properties and Salt Dependence into a Coarse-Grained Model of DNA. J. Chem. Phys. 2015, 142, 234901.

47. Pettersen, E. F.; Goddard, T. D.; Huang, C. C.; Couch, G. S.; Greenblatt, D. M.; Meng, E. C.; Ferrin, T. E. UCSF Chimera - a Visualization System for Exploratory Research and Analysis. J. Comput. Chem. 2004, 25, 1605-1612.

48. Gao, Y.; Wolf, L. K.; Georgiadis, R. M. Secondary Structure Effects on DNA Hybridization Kinetics: a Solution versus Surface Comparison. Nucleic Acids Res. 2006, 34, 3370-3377.

49. Schreck, J. S.; Ouldridge, T. E.; Romano, F.; Šulc, P.; Shaw, L.; Louis, A. A.; Doye, J. P. K. DNA Hairpins Destabilize Duplexes Primarily by Promoting Melting rather than by Inhibiting Hybridization. Nucleic Acids Res. 2015, 43, 6181-6190.

50. Genot, A. J.; Zhang, D. Y.; Bath, J.; Turberfield, A. J. Remote Toehold: a Mechanism for Flexible Control of DNA Hybridization Kinetics. J. Am. Chem. Soc. 2011, 133, 2177-2182.
51. Romano, F.; Hudson, A.; Doye, J. P. K.; Ouldridge, T. E.; Louis, A. A. The Effect of Topology on the Structure and Free-Energy Landscape of DNA Kissing Complexes. J. Chem. Phys. 2012, 136, 215102.

52. Yurke, B.; Turberfield, A. J.; Mills, A. P.; Simmel, F. C.; Neumann, J. A DNAFuelled Molecular Machine Made of DNA. Nature 2000, 406, 605-608.

53. Müller, B. K.; Reuter, A.; Simmel, F. C.; Lamb, D. C. Single-Pair FRET Characterization of DNA Tweezers. Nano Lett. 2006, $6,2814-2820$.

54. Tomov, T. E.; Tsukanov, R.; Liber, M.; Masoud, R.; Plavner, N.; Nir, E. Rational Design of DNA Motors: Fuel Optimization through Single-Molecule Fluorescence. J. Am. Chem. Soc. 2013, 135, 11935-11941.

55. Bae, W.; Kim, K.; Min, D.; Ryu, J.-K.; Hyeon, C.; Yoon, T.-Y. Programmed Folding of DNA Origami Structures through Single-Molecule Force Control. Nat. Comm. 2014, 5, 5654.

56. Douglas, S. M.; Marblestone, A. H.; Teerapittayanon, S.; Vazquez, A.; Church, G. M.; Shih, W. M. Rapid Prototyping of 3D DNA-Origami Shapes with caDNAno. Nucleic Acids Res. 2009, 37, 5001-5006.

57. Murtola, T.; Bunker, A.; Vattulainen, I.; Deserno, M.; Karttunen, M. Multiscale Modeling of Emergent Materials: Biological and Soft Matter. Phys. Chem. Chem. Phys. 2009, 11, 1869-1892. 